\title{
Article \\ Fluid-Dynamic Force Measurement of Ahmed Model in Steady-State Cornering
}

\author{
Takuji Nakashima $^{1, *}$, Hidemi Mutsuda ${ }^{1}{ }^{\circledR}$, Taiga Kanehira ${ }^{1}$ and Makoto Tsubokura ${ }^{2,3}$ \\ 1 Graduate School of Advanced Science and Engineering, Hiroshima University, 1-4-1 Kagamiyama, \\ Higashi-Hiroshima, Hiroshima 739-8527, Japan; mutsuda@hiroshima-u.ac.jp (H.M.); \\ taiga-kanehira@hiroshima-u.ac.jp (T.K.) \\ 2 RIKEN Center for Computational Science (R-CCS), 7-1-26 Minatojima-minami-machi, Chuo-ku, Kobe, \\ Hyogo 650-0047, Japan; mtsubo@riken.jp or tsubo@tiger.kobe-u.ac.jp \\ 3 Graduate School of System Informatics, Kobe University, 1-1, Rokkodai-cho, Nada-ku, Kobe-shi, \\ Hyogo 657-8501, Japan \\ * Correspondence: nakashima@hiroshima-u.ac.jp
}

Received: 20 November 2020; Accepted: 11 December 2020; Published: 14 December 2020

\begin{abstract}
The effects of on-road disturbances on the aerodynamic drag are attracting attention in order to accurately evaluate the fuel efficiency of an automobile on a road. The present study investigated the effects of cornering motion on automobile aerodynamics, especially focusing on the aerodynamic drag. Using a towing tank facility, measurements of the fluid-dynamic force acting on Ahmed models during steady-state cornering were conducted in water. The investigation included Ahmed models with slant angles $\theta=25^{\circ}$ and $35^{\circ}$, reproducing the wake structures of two different types of automobiles. The drag increase due to steady-state cornering motion was experimentally measured, and showed good agreement with previous numerical research, with the measurements conducted at a Reynolds number of $6 \times 10^{5}$, based on the model length. The Ahmed model with $\theta=35^{\circ}$ showed a greater drag increase due to the steady-state cornering motion than that with $\theta=25^{\circ}$, and it reached $15 \%$ of the total drag at a corner with a radius that was 10 times the vehicle length. The results indicated that the effect of the cornering motion on the automobile aerodynamics would be more important, depending on the type of automobile and its wake characteristics.
\end{abstract}

Keywords: aerodynamics; automobile; cornering; Ahmed model; drag increase; on-road condition; towing tank test

\section{Introduction}

In recent years, the aerodynamic drag performance of an automobile has become more important for its fuel efficiency because the efficiency of the powertrain has rapidly been improved through hybridization, electrification, and the improvement of combustion technology. In the conventional development process of vehicle aerodynamics, a vehicle subjected to a steady and uniform airflow in a wind tunnel or numerical simulation has been considered. This condition assumes a relative airflow acting on a vehicle running at a constant speed and a steady posture in stationary air. Additionally, the changes in the relative wind direction caused by on-road disturbance and their effects have been investigated using the so-called yaw condition or steady crosswind method [1], in which a real automobile [2] or an experimental vehicle model [3-5] is placed at a steady yaw angle with respect to a uniform flow. However, the impact of more realistic on-road disturbances on automobile aerodynamics has attracted attention to further increase the accuracy when evaluating the aerodynamic performance of an automobile on a road. For example, the on-road turbulence properties [6,7] and the effects of their fluctuation on the aerodynamic drag [8-10] have been investigated. The changes in the aerodynamic 
drag due to more specific disturbances from a passing vehicle [11] have also been reported. Moreover, vehicle motion, which is another type of on-road disturbance, also affects the aerodynamics. As a typical vehicle motion on a road, the effects of a cornering motion on the aerodynamics have been investigated [12-16].

Although vehicle cornering is accompanied by a complicated posture change, the main component other than the forward motion is the yaw rotation. Because of the yaw rotation, the yaw angle of the free-stream flow acting on the vehicle is distributed in the longitudinal direction of the vehicle [13]. This spatial distribution of the relative flow direction causes the aerodynamics to be different from those under the general yaw condition in a wind tunnel. This effect should be clarified to understand the vehicle aerodynamics during cornering. Keogh et al. [13] conducted a large-eddy simulation of a simplified vehicle body during cornering. They reported the cornering effects on the aerodynamics and clarified the related aerodynamic phenomena. The cornering effects increased the drag force and generated a side force toward the center of the corner, as well as a yaw moment that dampened the yaw rotation. Josefsson et al. [14] conducted a steady Reynolds-averaged Navier-Stokes (RANS) simulation of a sedan-type vehicle during steady-state cornering and indicated that the asymmetric geometry of the underbody affected the drag increase due to the cornering motion. Tsubokura et al. [15] and Okada et al. [16] numerically investigated the unsteady aerodynamic characteristics of a sedan-type vehicle during meandering motion, focusing on the responses of the side force and yaw moment in relation to the drivability of the automobile. These researchers adopted special numerical techniques using non-inertial reference frame and modified boundary condition [17] for vehicle aerodynamics to reproduce the cornering motion. However, they investigated the cornering effects on the aerodynamics for a specific vehicle geometry, with the shape variation in the research limited to details of the body shape [14] or aerodynamic parts [15,16]. To understand the effects more universally, it would be effective to investigate the effects on vehicles with different fundamental aerodynamic characteristics under the same cornering condition. A typical difference in the fundamental aerodynamic characteristics is a difference in the wake flow structure, which is generally classified as a notch-back, fast-back, or square-back type of flow in association with the classical rear end shapes [18].

Furthermore, it is difficult to conduct wind tunnel experiments that reproduce the effects of cornering motion because the streamlines of the relative airflow observed from the cornering vehicle are not straight but curved. When a curved test section is used to generate the curved free-stream, the flow velocity is distributed in the radial direction, and a pressure gradient balanced by the centrifugal force is also generated. Even when other special techniques are used, such as a bent model [19], which reproduces the effects of curved flow in a straight flow field, or a special wind tunnel with a rotating test section [20] designed to cancel out the radial pressure distribution, it is difficult to precisely and flexibly reproduce the relative flow acting on a cornering vehicle. On the other hand, a ship model basin for a towing test, which is generally used to measure the hydrodynamic force acting on a ship model, can measure the fluid-dynamic forces under steady-state cornering conditions, which is the so-called circular motion test (CMT) [21]. Such towing tank facilities can directly apply the cornering motion to a vehicle model, without requiring the Galilean transformation assumed in wind tunnel measurements. Moreover, because a towing tank uses water as the fluid, it has other advantages over a wind tunnel that uses air. Due to the lower dynamic viscosity, the similar flow at the same Reynolds number can be observed as a relatively slower phenomenon in the water tank. Due to the higher density of water, relatively larger fluid-dynamic force acts on the same length-scale model in the similar flow field, allowing for more accurate measurements. Therefore, some towing tank tests on automobile aerodynamics [22-25] have already been reported. When moving ground systems were not standard in automobile aerodynamics measurement, some studies on the ground effect of automobile aerodynamics have been conducted in a towing tank, taking the advantage of not requiring the Galilean transformation. Vorwaller and Germane [22] towed two race-car models with a length of $0.25 \mathrm{~m}$ to measure the drag force under the ground effect with rolling wheels. Larsson et al. [23] towed a real production automobile in a water basin to investigate the ground effect. They measured 
fluid-dynamic drag and lift forces and compared them with the wind tunnel measurement. From the comparisons, they reported a considerable over estimation of lift force in a wind tunnel with a ground simulation using upstream boundary layer suction, while the drag force in both facilities showed good agreement. Aoki et al. [24] conducted towing tests of a one-fifth-scale sedan-type automobile model in a ship model basin to measure the fluid-dynamic force and surface pressure acting on the automobile model under the ground effect, and to visualize the flow around the vehicle. Towing tank tests have also been used to measure the fluid-dynamic responses of automobile models with pitching motion [25]. Because the density of the experimental model can easily be close to the density of water, the inertial force due to the model acceleration becomes relatively smaller than that during wind tunnel measurements. This characteristic is also suitable for the fluid dynamics measurement of a vehicle during cornering motion because the cornering motion is an accelerating motion in the lateral direction of the vehicle. In our previous research [26,27], the fluid-dynamic force acting on a one-fifth-scale model of a sedan-type automobile during steady-state cornering was measured in a towing tank facility. These measurements clarified the characteristics of the side force and yaw moment, which were similar to those with a simplified model [13], focusing on the aerodynamic effects on the drivability. However, the effects of the cornering motion on the drag force were not experimentally measured and clarified. Moreover, the measurement was conducted on one vehicle model without geometric variation.

In research on automobile aerodynamics, the shapes of actual automobiles are too complicated to draw a universal conclusion. Therefore, fundamental research on automobile aerodynamics often uses a very simplified vehicle model that reproduces the essential characteristics of the flow around an automobile. The simplified model proposed by Ahmed et al. [28], the so-called Ahmed model or Ahmed body, is one of the most famous models in the automobile aerodynamics research field. As shown in Figure 1, this model has a smooth round head and a box-shaped body with a slant surface from the rear end of the roof to the top of the base. Only slant angle $\theta$ is a shape parameter that affects the aerodynamic characteristics of the model. In particular, at the critical slant angle of $30^{\circ}$, the drag and lift change drastically with the wake structure. The differences in the wake structure and the detailed characteristics of the flow field before and after the critical slant angle have been investigated using experimental measurements [28-31] and numerical analyses [32,33]. Slant angles $\theta=25^{\circ}$ and $\theta=35^{\circ}$ have often been adopted as the subcritical and postcritical conditions. The subcritical model with $\theta=25^{\circ}$ shows a highly three-dimensional flow (called a "three-dimensional separated (TDS) flow" [31] or "strongly three-dimensional wake" [32]), in which the flow separated at the leading edge of the slant surface reattaches to the surface. The post-critical flow field represented by $\theta=35^{\circ}$ has a pseudo two-dimensional flow structure (called a "quasi-axisymmetric- separated (QAS) flow" [31] or "quasi-two-dimensional wake" [32]), with uniformity in the lateral direction of the model without any reattachment of the flow onto the slanted surface. These wake structures can be regarded as fast-back and square-back flows in the classification of the wake structures of automobiles [18], respectively. Furthermore, Ahmed models with different wake structures have shown significantly different response characteristics to disturbances [1,4,34]. Regarding the response to a steady crosswind condition, only the postcritical model showed aerodynamic bi-stability under a certain yaw angle condition [4]. The responses of the wake velocity distribution and turbulence characteristics to a steady crosswind were also different between the sub- and post-critical models [1]. The aerodynamic responses to changes in the relative wind speed [34] also showed some different trends for the drag and lift behaviors between the sub- and post-critical models. These results implied the importance of considering the difference in the wake structure in research on the aerodynamic response of a vehicle to an on-road disturbance.

The purpose of this study was to experimentally measure the effects of a cornering motion on vehicles' aerodynamics, especially the aerodynamic drag, and to further understand their characteristics. For this purpose, fluid-dynamic force measurements were conducted on vehicle models during cornering using a towing tank facility to experimentally clarify the cornering effects. To clarify the universal characteristics of the cornering effects, simplified vehicle models with different wake 
characteristics were investigated. Ahmed models with different slant angles, for which the wake structures could be categorized as those of square-back and fast-back type automobiles, were chosen as the investigated vehicle models. To validate the measurement results, the influences of the submersion depth and towing speed were investigated, and the fluid-dynamic force was compared with that in previous numerical research [13]. Then, the cornering effects on the fluid-dynamic force were quantitatively clarified. This investigation focused on the differences in the effects between the two models, which should have depended on their different wake characteristics. Furthermore, the characteristics of the change in the fluid-dynamic force due to the cornering motion were also examined in comparison with a uniform crosswind condition. This investigation clarified the similarities and differences in the aerodynamic characteristics of a cornering vehicle compared to the steady crosswind aerodynamics that can be measured in general wind-tunnel tests.

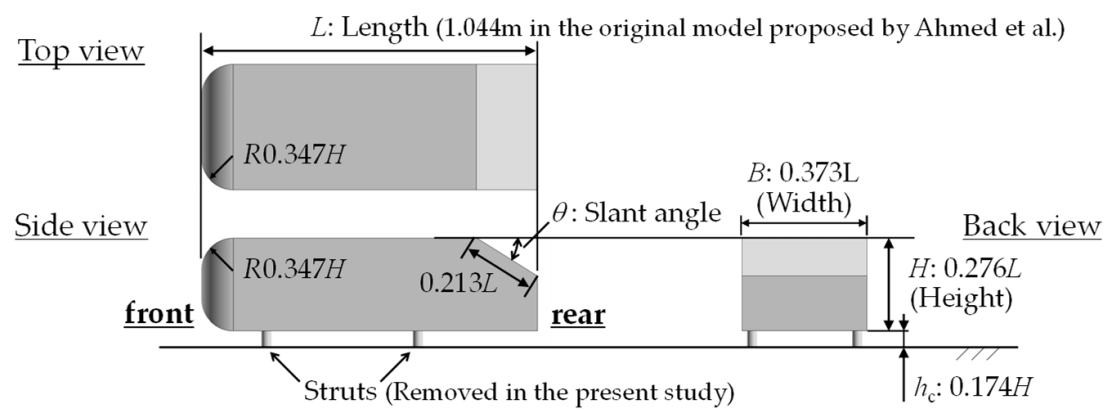

(a)

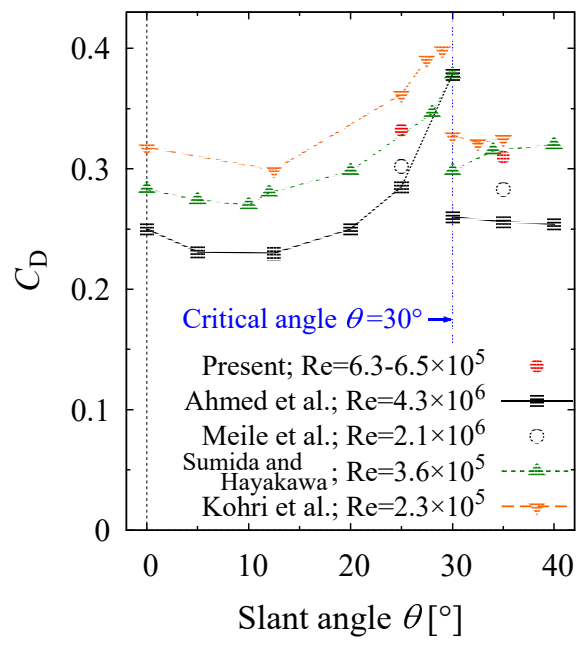

(b)

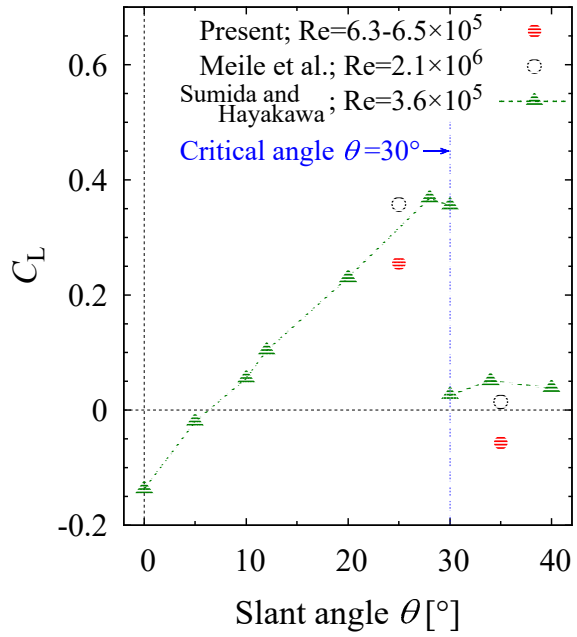

(c)

Figure 1. Simplified vehicle model proposed by Ahmed et al. [28] (so-called Ahmed model), and its aerodynamic characteristics depending on slant angle $\theta$ : (a) orthogonal view of model; (b) dependency of drag coefficient $C_{\mathrm{D}}$ on $\theta$ compared with Ahmed et al. [28], Meile et al. [4], Sumida and Hayakawa [34], and Kohri et al. [30]; and (c) dependency of lift coefficient $C_{\mathrm{L}}$ on $\theta$ compared with Meile et al. [4] and Sumida and Hayakawa [34].

\section{Methods}

\subsection{Investigated Vehicle Model and Vehicle Motion}

The adopted model was constructed using a scale that was four-fifths that of the original Ahmed model [28], which itself was an approximately $1 / 5$ scale model of an actual automobile. Figure 2 shows a schematic of the experimental model. The model had a length $(L)$ of $0.835 \mathrm{~m}$, width $(B)$ of $0.311 \mathrm{~m}$, and height $(H)$ of $0.230 \mathrm{~m}$. Under-body clearance $h_{\mathrm{c}}$ was set to $0.04 \mathrm{~m}$ to have the same ratio as the original model. Two slant angles $\left(\theta=25^{\circ}\right.$ and $\left.35^{\circ}\right)$ were used to reproduce the different wake 
structures for fast-back and square-back type automobiles [18], respectively. They were realized using exchangeable rear parts for the model. The four struts under the body in the original geometry were removed, and a circular rod covered by a part of the wing section was introduced to suspend the model from above.
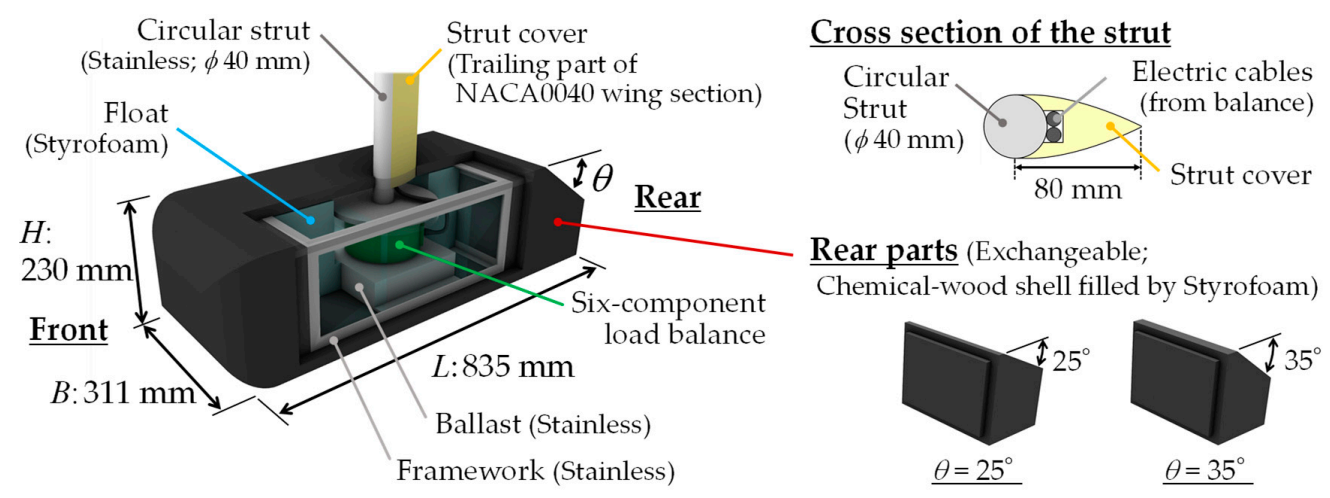

Figure 2. Schematic image of experimental model. The figure shows the dimensions, configuration, and inner structure of the model, with the side and top of the left half of the body translucent. The sub-figure on the upper right shows the cross-sectional geometry of the strut.

Figure 3 shows the definitions of the investigated vehicle motions and coordinate system fixed on the vehicle. One is the steady-state cornering motion, and the other is a side-slip motion simulating the steady crosswind condition. The steady-state cornering motion consisted of constant yaw rate $\omega$ at the center of the model and constant forwarding speed $U_{\mathrm{t}}$, which was given as the towing speed in the measurement, as shown in Figure 3a. The relationship between normalized yaw rate $\omega^{\prime}=\omega /\left(U_{\mathrm{t}} / L\right)$ and cornering radius $R=U_{\mathrm{t}} / \omega$ during the steady-state cornering motion can be derived as follows. Thus, normalized cornering radius $R^{\prime}=R / L$ becomes a reciprocal of $\omega^{\prime}$. For a discussion of a local fluid-dynamic response at the rear part of the model, the local yaw angle at the trailing edges of the model is defined as $\beta_{\mathrm{TE}}$.

$$
\omega^{\prime}=\omega /\left(U_{\mathrm{t}} / L\right)=\left(U_{\mathrm{t}} / R\right) /\left(U_{\mathrm{t}} / L\right)=(R / L)^{-1} .
$$

Based on yaw rate $\omega$ and distance $L / 2$ from the rotational center to the edges, $\beta_{\mathrm{TE}}$ during steady state cornering can be determined as follows:

$$
\beta_{\mathrm{TE}}=\tan ^{-1}\left\{-(L / 2) \omega / U_{\mathrm{t}}\right\} \approx-L \omega / 2 U_{\mathrm{t}}=-\omega^{\prime} / 2[\mathrm{rad}],
$$

assuming $\omega^{\prime}<<1$.

Another investigated vehicle motion is a straight towing motion with constant speed $U_{\mathrm{t}}$ and yaw angle $\beta$, as shown in Figure $3 \mathrm{~b}$. It reproduces the steady crosswind condition where the uniform relative flow acts on the model at yaw angle $\beta$. The $\beta_{\mathrm{TE}}$ value was the same as yaw angle $\beta$ at the model center because of the uniform relative flow.

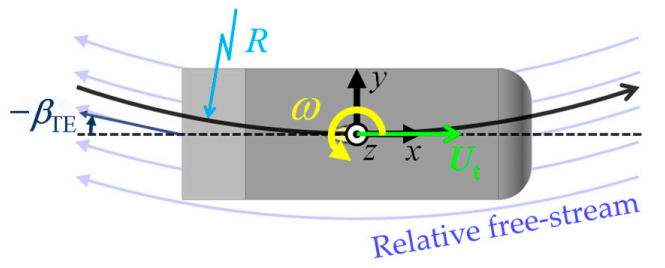

(a)

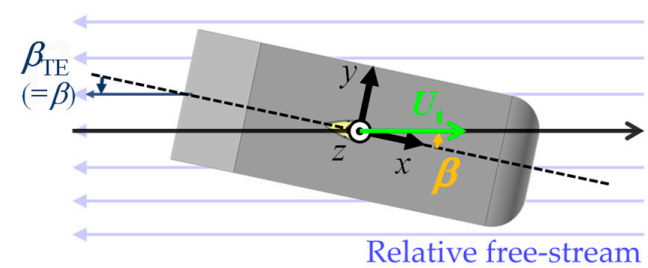

(b)

Figure 3. Definitions of vehicle motions and their components: (a) steady-state cornering motion parameterized by forwarding speed $U_{\mathrm{t}}$ and yaw rate $\omega$ at longitudinal center of model; and (b) steady crosswind condition parameterized by $U_{\mathrm{t}}$ and yaw angle $\beta$. In addition, definitions of a coordinate system $x-y-z$ fixed on the vehicle and the local yaw angle $\beta_{\text {LE }}$ at the trailing edge are also drawn. 
Here and after, the measurements for the steady-state cornering motion are called the results of the "circular motion test" (CMT) [21]. Moreover, the measurements under the steady crosswind condition and the straight towing measurements with a zero yaw angle are called the results of the "steady cross-wind test" (SCW) and "straight motion test" (SMT), respectively.

\subsection{Experimental Setup}

The fluid-dynamic force acting on the vehicle model during cornering and straight motions in water were measured in a towing tank facility at Hiroshima University. Figure 4 shows a schematic image of the towing tank facility and experimental setup. The facility consists of a water tank and towing carriage system traversing the tank. The water tank has a length of $100 \mathrm{~m}$, maximum width of $10 \mathrm{~m}$ (with a main section width of $8 \mathrm{~m}$ ), and depth of $3.6 \mathrm{~m}$. A lifting-type false bottom with a flat surface length of $25 \mathrm{~L}$ and width of $16 \mathrm{~B}$ is installed in the center of the water tank for shallow-water condition tests. The false bottom was set to depth $d_{\mathrm{f}}=4.5 \mathrm{H}$, which was the deepest limit of the equipment, and submersion depth $d_{\mathrm{s}}$ became $3.3 \mathrm{H}$. The vehicle model was towed above the false bottom to simulate the ground effect on its fluid dynamics. The towing carriage system consisted of two carriages. The main carriage traversed in the longitudinal direction of the tank. The maximum speed of the main carriage was $3.0 \mathrm{~m} / \mathrm{s}$. The sub-carriage traversed in the lateral direction of the tank on rails mounted on the main carriage, and it had a rotary actuator to rotate the experimental model about a vertical axis. The synchronous motion of these main and sub-carriages realized a steady-state cornering motion for the model in the horizontal plane. The motion was controlled by LabView in-house code, and the program simultaneously controlled an A/D converter to synchronously record the output signal from a load balance.

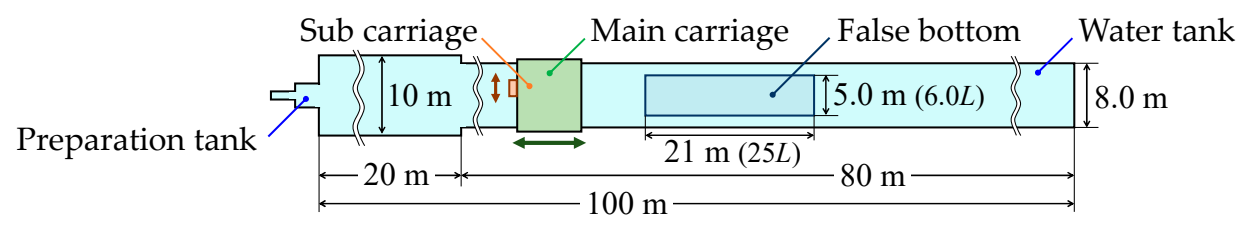

(a)

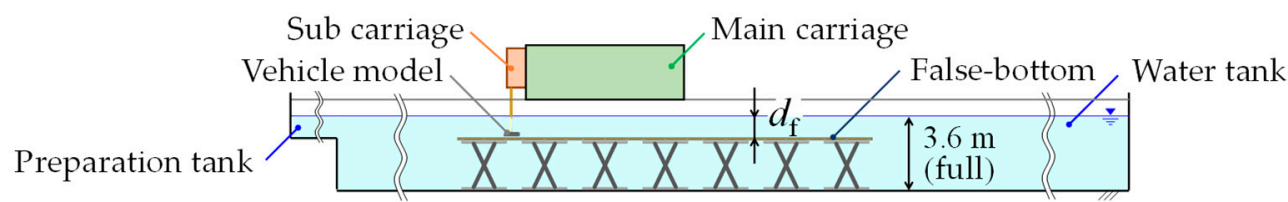

(b)

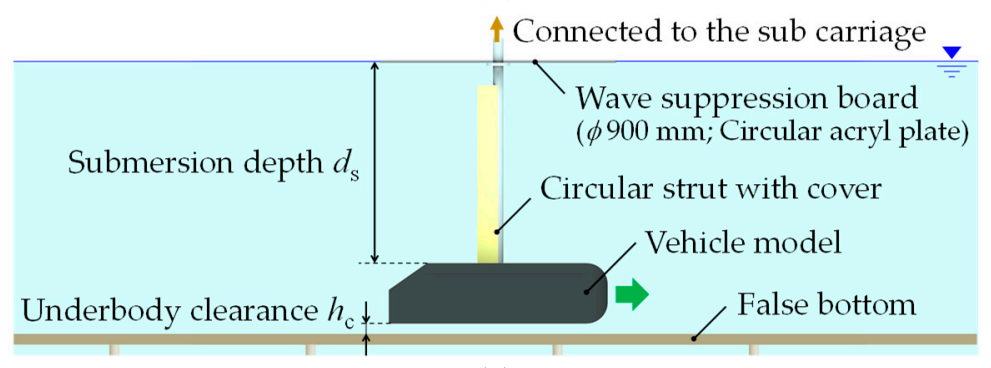

(c)

Figure 4. Schematic image of towing tank facility: (a) top view, (b) side view, and (c) magnified side view around experimental model.

The six components of the fluid-dynamic force and moment acting on the vehicle model were measured using a waterproof six-component load balance (NISSHO-ELECTRIC-WORKS; LMC-6566A-200NWP) installed inside the model. The maximum load of the load balance was $200 \mathrm{~N}$ for the horizontal force components $\left(F_{x}\right.$ and $\left.F_{y}\right), 400 \mathrm{~N}$ for the vertical force component $\left(F_{z}\right)$, 
and $20 \mathrm{Nm}$ for all three moments $\left(M_{x}, M_{y}\right.$, and $\left.M_{z}\right)$. Based on the results of the calibration test at the full scale of the load, the maximum hysteresis and non-linearity of each force component were less than $0.10 \mathrm{~N}$ and $0.046 \mathrm{~N}$, respectively. These errors corresponded to force coefficients of 0.0045 and 0.0020 , respectively, at a towing speed of $0.80 \mathrm{~m} / \mathrm{s}$. In the same evaluation for the moment errors, the summation of the maximum hysteresis and non-linearity was $7 \times 10^{-4}$ in terms of the moment coefficients. The interference between the six components was linearly corrected using a correction matrix. As shown in Figure 2, the balance was supported by a stainless circular rod that penetrated the roof of the model with $2 \mathrm{~mm}$ of clearance to measure the fluid-dynamic force acting on the model, except for the strut. The circular rod was connected to the sub-carriage of the towing tank facility, and electric cables from the load balance were connected to an amplifier through the inside of a rod cover, which had the cross-sectional shape of the trailing part of the NACA0040 wing section. The strain output from the load balance was amplified by a dynamic strain amplifier (IZUMISOKKI; DA-18K) and recorded by a 16-bit A/D converter (NI; PCIe-6321). In the CMT, the sampling rate was set to $20 \mathrm{~Hz}$, similar to the control rate of the towing carriage, and the data were recorded with the carriage motion simultaneously. The output signal was filtered by a $10 \mathrm{~Hz}$ low pass filter at the amplifier prior to the A/D conversion. Under the other measurement conditions, the output signal was filtered by $30 \mathrm{~Hz}$ low pass filter and sampled at $100 \mathrm{~Hz}$.

Figure 5 shows the definition of the coordinate system for the fluid-dynamic force and moment components. In the CMT, centrifugal side force $F_{y, i}$ acted on the experimental model. This was the inertial force due to the cornering motion and depended on its mass $m$, towing speed $U_{t}$, and yaw rate $\omega . F_{y, i}$ was removed from measured side force $F_{y, m}$ to extract fluid-dynamic side force $F_{y}$, as follows:

$$
F_{y}=F_{y, m}-F_{y, i}=F_{y, m}-m U_{t} \omega
$$

The yaw moment, $M_{z, i}$, caused by this centrifugal force was also considered. $M_{z, i}$ was estimated and removed from measured yaw moment $M_{z, m}$ to obtain fluid-dynamic yaw moment $M_{z}$, as follows:

$$
M_{z}=M_{z, m}-M_{z, i}=M_{z, m}-x_{c g} F_{y, I}=M_{z, m}-x_{c g} m U_{t} \omega,
$$

where $x_{c g}$ is the longitudinal distance between the center of gravity and the center of rotation. The mass of the model, $m$, including the mass of the water filling the inner space of the model, was measured by a weight scale, and it was $37.6 \mathrm{~kg}$. It generated a centrifugal force corresponding to 0.118 of the side force coefficient at the maximum yaw rate condition, $\omega^{\prime}=0.10$, and it was $150 \%$ of the fluid-dynamic side force in the case of $\theta=25^{\circ}$. To determine $x_{\mathrm{cg}}$, the experimental model was towed in air with the same motion as the CMT at $\omega^{\prime}=0.10$. Assuming that the aerodynamic force and moment were negligible, $x_{c g}$ was estimated as the ratio of the yaw moment to the lateral force, $M_{z, m} / F_{y, m}$, in the measurement. It was estimated that $x_{c g} / L=+0.009$. Additionally, the model weight estimated from the measured lateral force in the air was in good agreement with the directly measured weight, with $0.5 \%$ error.

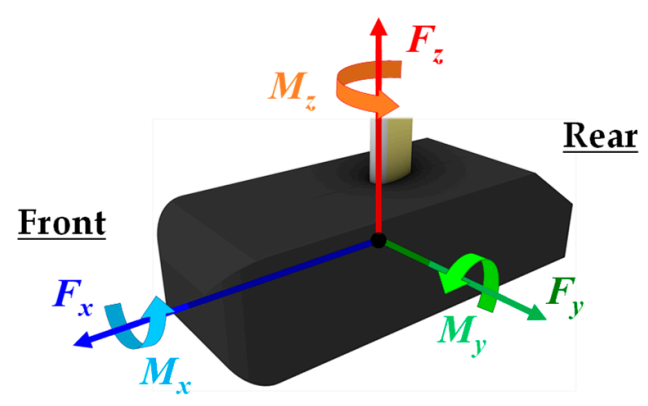

Figure 5. Definition of the coordinate system for the fluid-dynamic force and moment components.

Fluid-dynamic drag force $-F_{x}$, side force $F_{y}$, lift force $F_{z}$, and yaw moment $M_{z}$ were normalized as the force and moment coefficients: 


$$
C_{\mathrm{D}}=\frac{-2 F_{x}}{\rho U_{\mathrm{t}}^{2} A_{\mathrm{f}}}, \quad C_{\mathrm{S}}=\frac{2 F_{y}}{\rho U_{\mathrm{t}}^{2} A_{\mathrm{f}}}, \quad C_{\mathrm{L}}=\frac{2 F_{z}}{\rho U_{\mathrm{t}}^{2} A_{\mathrm{f}}}, \text { and } C_{\mathrm{YM}}=\frac{2 M_{z}}{\rho U_{\mathrm{t}}^{2} A_{\mathrm{f}} L},
$$

respectively. Here, $\rho, U_{\mathrm{t}}$, and $A_{\mathrm{f}}$ are the density of the water, towing speed, and frontal area of the model, respectively.

\subsection{Measurement Conditions}

The conditions of normalized yaw rate $\omega^{\prime}$ in the CMT and yaw angle $\beta$ in the SCW are listed in Table 1. The lateral acceleration of a passenger car under normal driving conditions is up to $4.0 \mathrm{~m} / \mathrm{s}^{2}$ [35]. Assuming $13.9 \mathrm{~m} / \mathrm{s}(50 \mathrm{kph}$ ) for the running speed and $4.8 \mathrm{~m}$ for the vehicle length, the centrifugal force due to steady-state cornering motion reached $4.0 \mathrm{~m} / \mathrm{s}^{2}$ at cornering radius $R=48 \mathrm{~m}$, which corresponded to normalized yaw rate $\omega^{\prime}=0.10$. When $\omega^{\prime}$ was 0.10 , the local yaw angle at the rear end of the model, $\beta_{\mathrm{TE}}$, became $2.9^{\circ}$ ( $=0.05 \mathrm{rad}$ ). Therefore, the maximum $\omega^{\prime}$ and $\beta$ values were set at 0.10 and $3.0^{\circ}$, respectively.

Table 1. Measurement conditions of yaw rate $\omega^{\prime}$ for circular motion test (CMT) and yaw angle $\beta$ for steady cross-wind test (SCW).

\begin{tabular}{ccc}
\hline Test Series & Parameter & Value \\
\hline $\mathrm{CMT}$ & $\omega^{\prime}\left(=1 / R^{\prime}\right)[-]$ & $0, \pm 0.0067, \pm 0.013, \pm 0.020^{*}, \pm 0.033^{*}, \pm 0.048, \pm 0.067, \pm 0.1$ \\
$\mathrm{SCW}$ & $\beta\left[^{\circ}\right]$ & $0, \pm 0.25, \pm 0.50^{\#}, \pm 0.75, \pm 1.0, \pm 1.5^{\#}, \pm 2.0^{\# \#}, \pm 2.5^{\# \#}, \pm 3.0$ \\
\hline
\end{tabular}

$* \omega^{\prime}=+0.020, \pm 0.033$ for the model with $\theta=25^{\circ}$ were measured once more than the standard. ${ }^{\#} \beta=-0.5,-1.5$, for the model with $\theta=35^{\circ}$ were measured once more than the standard. $\#= \pm 2.0^{\circ}$ for both models, and at $\beta=2.5^{\circ}$ for the model with $\theta=35^{\circ}$ were measured three times more than the standard.

Regarding the number of measurements, 47 and 41 SMT measurements were conducted for the models with $\theta=25^{\circ}$ and $\theta=35^{\circ}$, respectively. For the CMT, the standard numbers of measurements were set at six and three for each positive and negative $\omega^{\prime}$, respectively. For the SCW, the standard number of measurements at each yaw angle on both sides was set at three. Under some of the measurement conditions, additional measurements were conducted once or three times. They are described by the superscript marks and footnote in Table 1. Additional measurements for the validation in Section 3.1, the measurements in each condition were conducted at least three times.

The measurement results were summarized by assuming symmetricity in the width direction of the model because the all fluid-dynamic coefficients showed approximately symmetric behaviors in that direction. The drag and lift forces were summarized using the absolute values of $\omega^{\prime}$ and $\beta$. Concerning the side force and yaw moment, not the absolute values of $C_{\mathrm{S}}$ and $C_{\mathrm{YM}}$, but their changes from the straight motion condition $\left(\Delta C_{S}\right.$ and $\left.\Delta C_{Y M}\right)$ were inverted and added. The variability of the measurement results under each condition was statistically determined, and the $95 \%$ confidence interval was estimated using Student's t-distribution. The confidence intervals are displayed as error bars for all the data plots. Then, the dependencies of the results on $\omega^{\prime}$ and $\beta$ in the CMT and SCW were fitted to polynomials of $\omega^{\prime}$ and $\beta$, respectively. Second-order polynomials were adopted to fit drag and lift coefficients $C_{\mathrm{D}}$ and $C_{\mathrm{L}}$. Changes in the side-force and yaw-moment coefficients $\Delta C_{\mathrm{S}}$ and $\Delta C_{\mathrm{YM}}$ were fitted to linear functions. The fitting was performed using Gnuplot 5.2 software.

In each measurement, after recording the zero point of the balance for $15 \mathrm{~s}$, the model was towed and accelerated to the steady state under each measurement condition. In the SMT and SCW, towing started before the front of the false bottom. A run-up section with a length of $10 \mathrm{~L}$ was taken after the model entered above the false bottom. Then, the fluid-dynamic forces and moments were measured and averaged in the $12 \mathrm{~L}$ long measurement section. In the CMT, the towing started above or near the front of the false bottom after recording the zero point of the balance. Figure 6 shows the towing trajectory of the CMT. As shown in the figure, the towing area was limited to a width of $3.6 \mathrm{~L}$ to avoid the effects of the false bottom edges. Because of the limitation of the arc height of the trajectory, the towing distance was shortened at higher values for $\omega^{\prime}$. When $\omega^{\prime}$ was less than 
0.048 , the length of the run-up section, including the acceleration to the steady state, was $11 \mathrm{~L}$, and the length of the measurement section was $12 L$. In the cases at higher $\omega^{\prime}$, the lengths of the run-up and measurement sections were $10 \mathrm{~L}$ and $3.7 \mathrm{~L}$ at $\omega^{\prime}=0.10 ; 10 \mathrm{~L}$ and $7.3 \mathrm{~L}$ at $\omega^{\prime}=0.067$; and $10 \mathrm{~L}$ and $11 \mathrm{~L}$ at $\omega^{\prime}=0.048$, respectively.

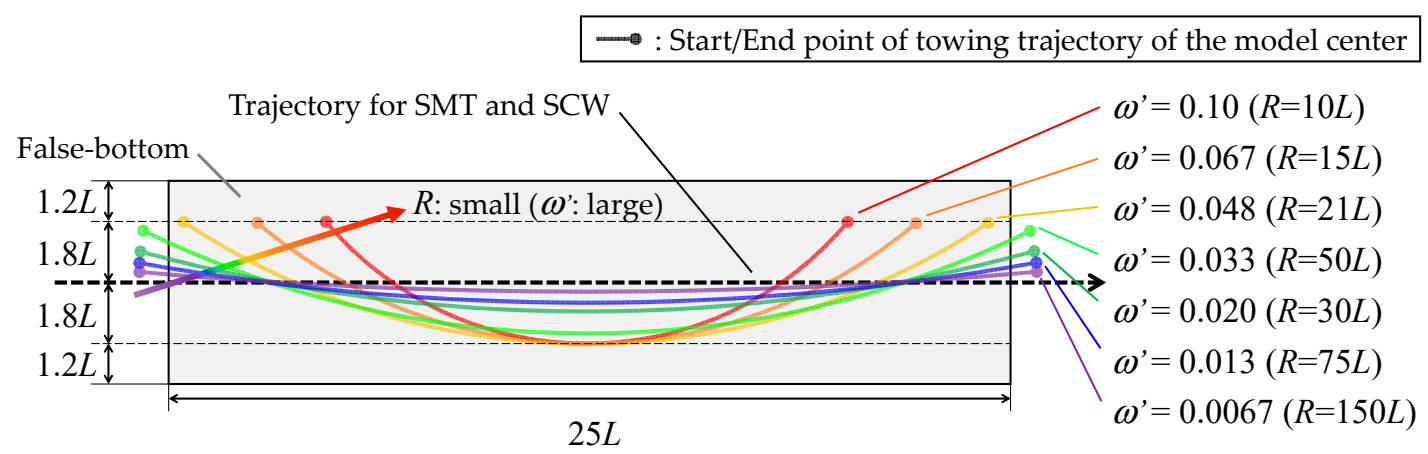

Figure 6. Top view of towing trajectory for CMT with each yaw rate (and cornering radius). The figure also shows the location of the false bottom and trajectory for straight motion test (SMT) and SCW.

The constant towing speed, $U_{\mathrm{t}}$, of the model was set at $0.8 \mathrm{~m} / \mathrm{s}$. The Reynolds numbers $(R e)$ based on the model length were $6.45 \times 10^{5}\left( \pm 0.06 \times 10^{5}\right)$ and $6.25 \times 10^{5}\left( \pm 0.03 \times 10^{5}\right)$ in the measurements of the models with $\theta=25^{\circ}$ and $\theta=35^{\circ}$, respectively. The wake flow characteristics of the Ahmed model showed less Reynolds number dependency in a range above $10^{5}$, although the drag force quantitatively changed because of the change in surface friction [30]. Thus, this study adopted an approach to reduce the Reynolds number, which has often been adopted in previous research on the aerodynamics of Ahmed models, such as [1,13,30,34]. Moreover, according to Aoki et al. [24], Froude number $F r_{\mathrm{d}}$, based on submersion depth $d_{s}$ of the model was defined as follows:

$$
F r_{\mathrm{d}}=\frac{U_{\mathrm{t}}}{\sqrt{g d_{\mathrm{s}}}}
$$

and had to be less than 0.37 to avoid the free-surface influence due to wave making. Here, $g$ is the acceleration of gravity. This criterion corresponded to an upper limit of towing speed $U_{\mathrm{t}}$ of $1.0 \mathrm{~m} / \mathrm{s}$ at the present submersion depth condition of $d_{\mathrm{s}}=3.3 \mathrm{H}$, and the towing speed was determined by taking a margin to this limit. Finally, Froude number $F r_{\mathrm{d}}$ was 0.29 at $U_{\mathrm{t}}=0.8 \mathrm{~m} / \mathrm{s}$. Additionally, the present submersion depth, $d_{\mathrm{s}}=3.3 \mathrm{H}$, satisfied the criterion of $d_{\mathrm{s}} / H>2$, as reported by Aoki et al. [24], to avoid the free-surface influence due to the blockage effect. The effects of the Reynolds number, Froude number, and submersion depth of the model were also investigated by changing the towing speed and depth of the water tank. The results are described in Section 3.1.

\section{Results}

As an example of the data measured in a single towing test, Figure 7 shows the time-series of the fluid-dynamic forces and moment measured in a CMT of the model with $\theta=35^{\circ}$ at $\omega^{\prime}=0.067$. It also shows the time series of $U_{\mathrm{t}}$ and $\omega^{\prime}$ associating with the towing trajectory of the model in Figure 7a. The thick line of each coefficient means the profile moving-averaged over $1.0 \mathrm{~s}$ in Figure $7 \mathrm{~b}, \mathrm{c}$. After accelerating and running in steady-state cornering motion for the run-up distance, the measured forces and moment were time-averaged during the measurement period, and then the model decelerated and stopped at the end of the towing test. 


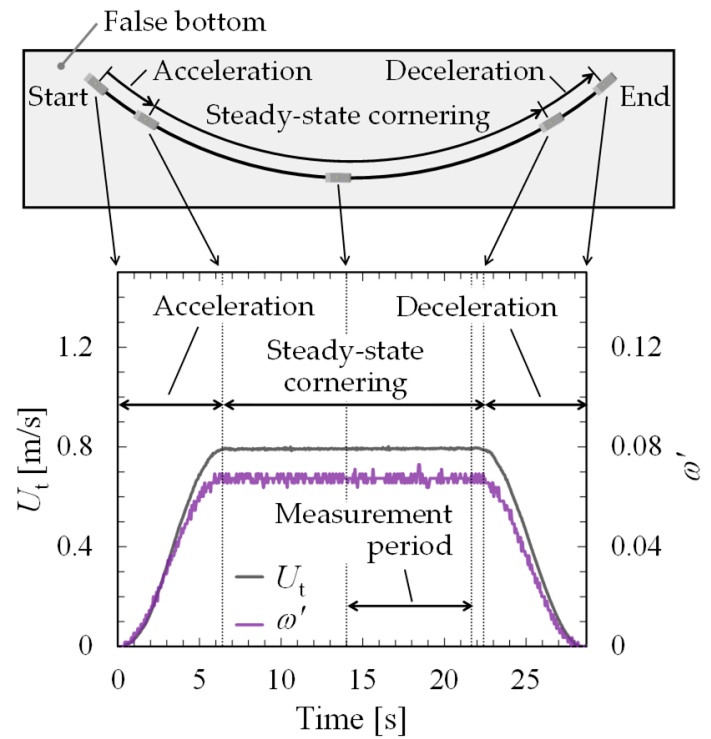

(a)

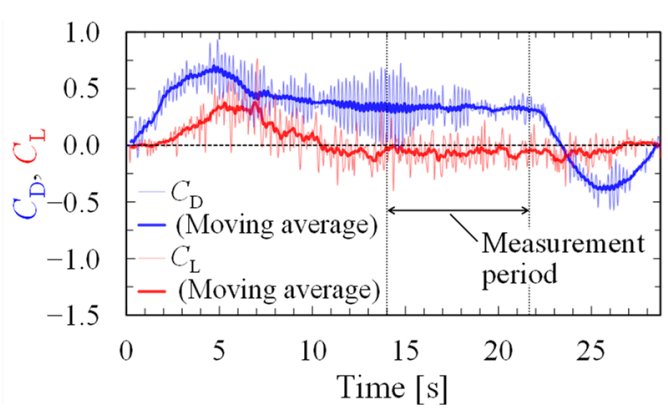

(b)

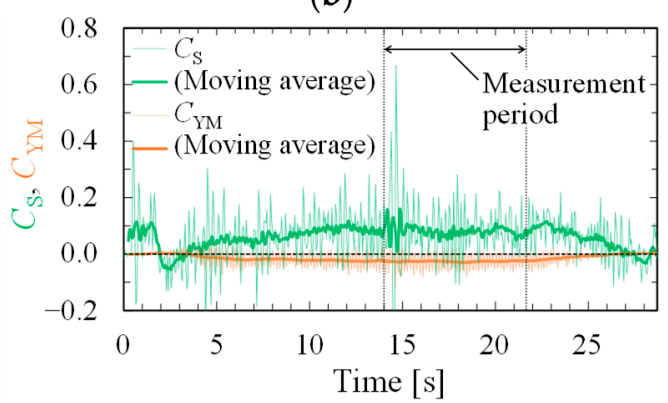

(c)

Figure 7. Time-series of vehicle motion and fluid-dynamic force measured in CMT with $\theta=35^{\circ}$ at $\omega^{\prime}=0.067$ : (a) towing speed $U_{\mathrm{t}}$ and normalized yaw rate $\omega^{\prime}$ (bottom) with a top view of the towing trajectory (top); (b) drag, and lift coefficients, $C_{\mathrm{D}}$ and $C_{\mathrm{L}} ;(\mathbf{c})$ side force and yaw moment coefficients, $C_{S}$ and $C_{Y M}$. The coefficients were calculated using a constant towing speed of $0.8 \mathrm{~m} / \mathrm{s}$ rather than the instantaneous $U_{\mathrm{t}}$. The thick line of each coefficient means the profile moving-averaged over $1.0 \mathrm{~s}$.

\subsection{Validation of Measurement Results}

Before the main measurements of the CMT and SCW, the measurement results were validated. First, the drag and lift force differences between the two Ahmed models with $\theta=25^{\circ}$ and $\theta=35^{\circ}$ were compared with the results of previous studies to confirm the reproduction of the fluid-dynamic characteristics. Then, to evaluate the influence of the test conditions on the measurement results, the dependency of the fluid-dynamic force on the submersion depth and towing speed was clarified. Finally, to quantitatively validate the results of the CMT, the changes in the fluid-dynamic coefficients of the Ahmed model with $\theta=25^{\circ}$ due to the steady state cornering motion were compared with the numerical results by Keogh et al. [13].

\subsubsection{Slant Angle Dependency}

The slant angle dependency of the fluid-dynamic drag and lift in the SMT was compared with that in previous studies $[4,28,30,34]$, and the reproduction of the essential fluid dynamics of the Ahmed models with $\theta=25^{\circ}$ and $\theta=35^{\circ}$ was confirmed, as displayed in Figure $1 \mathrm{~b}$,c. Table 2 also shows comparisons of the $C_{\mathrm{D}}$ and $C_{\mathrm{L}}$ values of the models with $\theta=25^{\circ}$ and $\theta=35^{\circ}$. The variations in $C_{\mathrm{D}}$ and $C_{\mathrm{L}}$ between the models with $\theta=25^{\circ}$ and $\theta=35^{\circ}$ show good agreement with the previous studies, while the absolute values of each $C_{D}$ and $C_{L}$ were quantitatively different as a result of the differences in the measurement conditions, especially in the Reynolds number shown in the second column of Table 2. According to Kohri et al. [30], the wake flow characteristics and base pressure have less Reynolds number dependency even at a lower Reynolds number of $2.3 \times 10^{5}$. Thus, the difference in $C_{D}$ depending on the Reynolds number was mainly caused by the change in the friction drag. Moreover, the drag coefficient of the model with $\theta=25^{\circ}$ in the present study showed reasonable agreement with an estimated drag coefficient at the same Reynolds number by the approximate function derived by Meile et al. [4]. Although the present $C_{D}$ value was still 0.014 smaller than the estimated one, the removal of four struts may have caused the difference. Concerning the lift force, lift coefficients 
that were approximately 0.1 smaller than those in previous studies $[4,34]$ were also reasonable because of the different conditions under the body. The ground surface was not static but moving relatively in the present study, which could have decreased the pressure on the bottom of the body as a result of the higher flow rate under the body. This tendency corresponds to the conclusion of Larsson et al. [23], in which the higher lift force under a fixed ground condition in a wind tunnel than a moving ground condition in a towing tank. Therefore, the slant angle dependencies of the drag and lift coefficients were verified, and the reproduction of the essential fluid dynamics of the Ahmed models with $\theta=25^{\circ}$ and $\theta=35^{\circ}$ was validated.

Table 2. Comparison of slant angle dependencies of $C_{\mathrm{D}}$ and $C_{\mathrm{L}}[4,28,30,34]$.

\begin{tabular}{cccccccc}
\hline & \multirow{2}{*}{$\begin{array}{c}\text { Reynolds No. } \\
\operatorname{Re} \times \mathbf{1 0}^{-\mathbf{6}}\end{array}$} & \multicolumn{3}{c}{ Drag Coefficient $C_{\mathrm{D}}$} & \multicolumn{3}{c}{ Lift Coefficient $C_{\mathrm{L}}$} \\
\cline { 3 - 8 } & $\boldsymbol{\theta}=\mathbf{2 5 ^ { \circ }}$ & $\boldsymbol{\theta}=\mathbf{3 5 ^ { \circ }}$ & Diff. & $\boldsymbol{\theta}=\mathbf{2 5 ^ { \circ }}$ & $\boldsymbol{\theta}=\mathbf{3 5 ^ { \circ }}$ & Diff. \\
\hline Present study & $0.63-0.65$ & 0.333 & 0.310 & 0.023 & 0.255 & -0.057 & -0.312 \\
\hline Ahmed et al. [28] & 4.29 & 0.29 & 0.26 & 0.03 & N.A. & N.A. & N.A. \\
\hline Meile et al. [4] & 2.05 & 0.30 & 0.28 & 0.02 & 0.36 & 0.01 & -0.35 \\
& 0.69 & 0.34 & N.A. & N.A. & 0.34 & N.A. & N.A. \\
\hline Sumida and & 0.65 & $0.347^{+}$ & N.A. & N.A. & N.A. & N.A. & N.A. \\
\hline Hayakawa [34] & 0.36 & $0.33^{*}$ & $0.32^{* *}$ & 0.01 & $0.32 *$ & $0.05 * *$ & -0.27 \\
\hline Kohri et al. [30] & 0.23 & 0.36 & 0.33 & 0.03 & N.A. & N.A. & N.A. \\
\hline
\end{tabular}

${ }^{\dagger}$ Estimated value from an approximate function of Reynolds number dependency of $C_{\mathrm{D}}$ [4]. ${ }^{*}$ Estimated value from linear interpolation between the values of $\theta=20^{\circ}$ and $\theta=28^{\circ}$. ${ }^{* *}$ Estimated value from linear interpolation between the values of $\theta=34^{\circ}$ and $\theta=40^{\circ}$.

\subsubsection{Submersion Depth Dependency}

Regarding the effects of a blockage in the test section and wave-making of the model on the measurement results, their dependency on submersion depth $d_{\mathrm{s}}$ of the model and the validity of the condition of $d_{\mathrm{s}}$ were investigated. The fluid-dynamic forces acting on the model with $\theta=35^{\circ}$ in the SMT and CMT at $\omega^{\prime}=0.067$ were measured under some submersion depth conditions, as shown in Table 3. Figure 8 shows the dependencies of $C_{\mathrm{D}}$ and $C_{\mathrm{L}}$ on normalized submersion depth $d_{\mathrm{s}} / H$. The lift converged to a constant in $d_{\mathrm{S}} / H>2.0$, as reported by Aoki et al. [24]. Though the drag was still slightly decreasing at standard depth $d_{\mathrm{s}} / H=3.3$, the variation was within a range of 0.01 . In addition, the similar convergence of $C_{\mathrm{D}}$ and $C_{\mathrm{L}}$ in the SMT has also been confirmed in the model with $\theta=25^{\circ}$, though it was measured under different conditions due to a different water temperature. Moreover, the dependencies of the changes in the coefficients of drag $\Delta C_{\mathrm{D}}$, lift $\Delta C_{\mathrm{L}}$, side-force $\Delta C_{\mathrm{S}}$, and yaw-moment $\Delta C_{\mathrm{YM}}$ due to the cornering motion at $\omega^{\prime}=0.067$ in Figure $8 b, c$ showed less sensitivity to the submersion depth. The variation in the change in each coefficient was smaller than its confidence interval. Therefore, the submersion depth did not to affect the conclusions about the effects of the cornering motion, and the condition was validated.

Table 3. Measurement conditions to investigate effect of submersion depth.

\begin{tabular}{ccccc}
\hline Test Series & Model & Submersion Depth $\boldsymbol{d}_{\mathbf{s}}$ & Froude No. $\boldsymbol{r}_{\mathbf{d}}$ & Reynolds No. Re \\
\hline & & $0.29 \mathrm{~m}(1.3 H)$ & 0.47 & \\
Straight motion, & & $0.42 \mathrm{~m}(1.8 H)$ & 0.39 & $6.25 \times 10^{5}$ \\
CMT $\left(\omega^{\prime}= \pm 0.067\right)$ & $\theta=35^{\circ}$ & $0.54 \mathrm{~m}(2.3 H)$ & 0.35 & $\left( \pm 0.02 \times 10^{5}\right)$ \\
& & $0.65 \mathrm{~m}(2.8 H)$ & 0.31 & \\
\hline
\end{tabular}

* The standard conditions of the present study. 


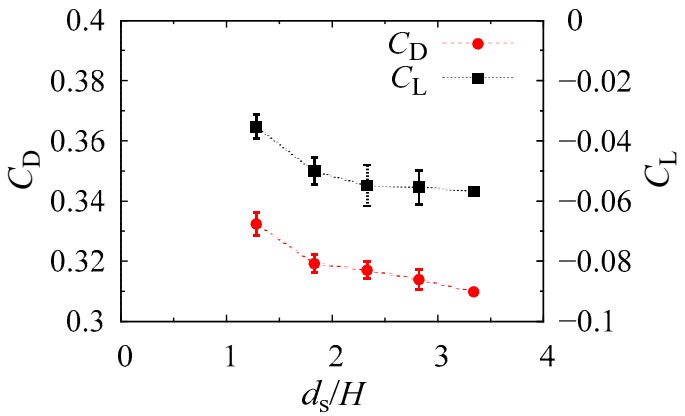

(a)

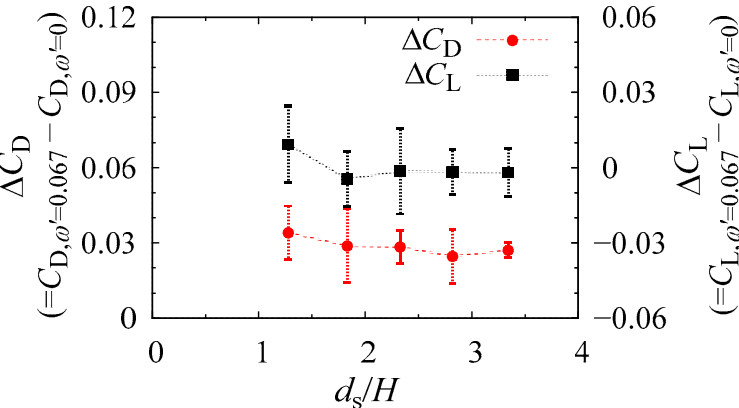

(b)

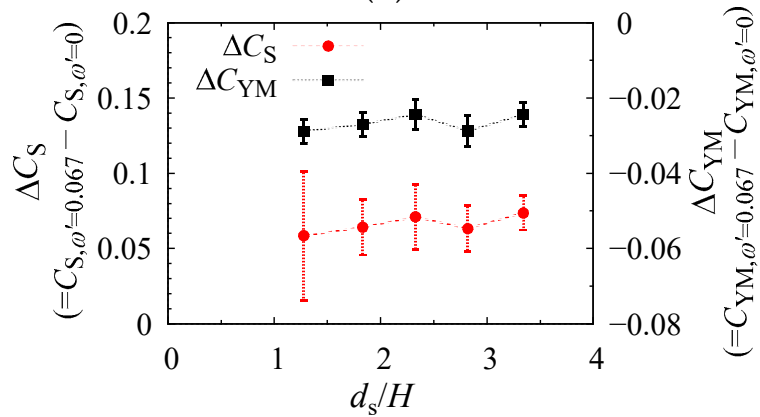

(c)

Figure 8. Depth dependency of fluid-dynamic characteristics of model with $\theta=35^{\circ}$ : (a) drag and lift coefficients $C_{\mathrm{D}}$ and $C_{\mathrm{L}} ;(\mathbf{b})$ changes in drag and lift coefficients in CMT at $\omega^{\prime}=0.067$ from SMT $\Delta C_{\mathrm{D}}$ and $\Delta C_{\mathrm{L}} ;(\mathbf{c})$ like $(\mathbf{b})$, changes in side force and yaw moment coefficients $\Delta C_{\mathrm{S}}$ and $\Delta C_{\mathrm{YM}}$.

\subsubsection{Towing Speed Dependency}

To validate the condition of $U_{\mathrm{t}}$, the dependency of the fluid-dynamic force on towing speed $U_{\mathrm{t}}$ was also investigated using the model with $\theta=35^{\circ}$ in the SMT. The measurement conditions are summarized in Table 4. Figure 9 shows the dependencies of $C_{\mathrm{D}}$ and $C_{\mathrm{L}}$. Here, Reynolds number $R e$ is adopted as the horizontal axis of the graph representing the variation of $U_{\mathrm{t}}$. When $U_{\mathrm{t}}$ increased, both the Froude and Reynolds numbers increased, and they generated opposite effects on $C_{\mathrm{D}}$ because of increasing the wave-making and decreasing the friction drag, respectively. Because they partially canceled each other out, it was difficult to determine the high-Reynolds number limit from this measurement. The $C_{\mathrm{D}}$ graph includes a fitted curve derived by Meile et al. [4], although it is not for $\theta=35^{\circ}$ but for $\theta=25^{\circ}$. The $C_{D}$ value shows a similar decreasing gradient to the fitted curve for $\theta=25^{\circ}$ around the standard measurement condition, $R e=6.45 \times 10^{5}$, while there is quantitative difference among them due to the difference of slant angle $\theta$. This trend is reasonable because the pressure drag acting on the base of the Ahmed model had less sensitivity to the Reynolds number [30], and the Reynolds number dependency of $C_{\mathrm{D}}$ may have mainly been caused by the change in the friction drag. It also indicates that the Reynolds number effect was dominant rather than the wave-making effect under the standard measurement condition. On the other hand, at the maximum towing speed at $R e=1.01 \times 10^{6}, F r_{\mathrm{d}}$ becomes 0.47, which exceeds the criterion by Aoki et al. [24]. Thus, the decreasing friction drag with increasing Re may be partially canceled by the wave-making drag, and the decrease in $C_{\mathrm{D}}$ from $R e=7.53 \times 10^{5}$ to $R e=1.01 \times 10^{6}$ may become smaller. Furthermore, the $C_{\mathrm{L}}$ in Figure $8 \mathrm{~b}$ has already converged to a constant at $R e=6.45 \times 10^{5}$. From these results, the standard condition of towing speed $U_{\mathrm{t}}$ was valid to investigate the drag increase due to cornering, where the change in the pressure drag was dominant [13]. 
Table 4. Measurement conditions to investigate effect of towing speed.

\begin{tabular}{ccccc}
\hline Test Series & Model & Towing Speed $U_{\mathbf{t}}$ & Froude No. $F r_{\mathbf{d}}$ & Reynolds No. $\boldsymbol{R} \boldsymbol{e}$ \\
\hline \multirow{3}{*}{ Straight } & \multirow{2}{*}{$\theta=35^{\circ}$} & $1.3 \mathrm{~m} / \mathrm{s}$ & 0.47 & $1.01 \times 10^{6}$ \\
& & $1.0 \mathrm{~m} / \mathrm{s}$ & 0.35 & $7.53 \times 10^{5}$ \\
& & $0.8 \mathrm{~m} / \mathrm{s}^{*}$ & 0.29 & $6.25 \times 10^{5}$ \\
& & $0.7 \mathrm{~m} / \mathrm{s}$ & 0.24 & $5.18 \times 10^{5}$ \\
\hline
\end{tabular}

* The standard conditions of the present study.

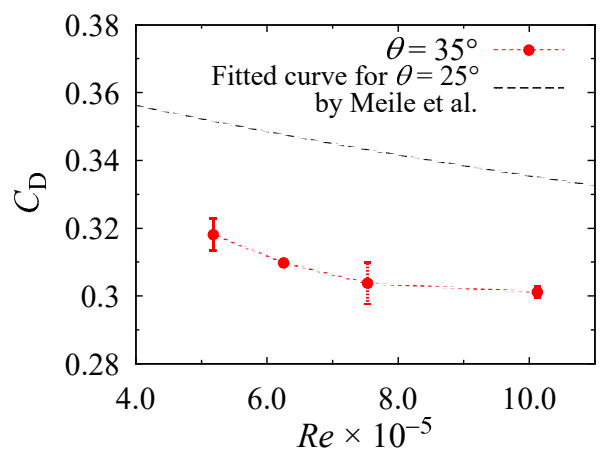

(a)

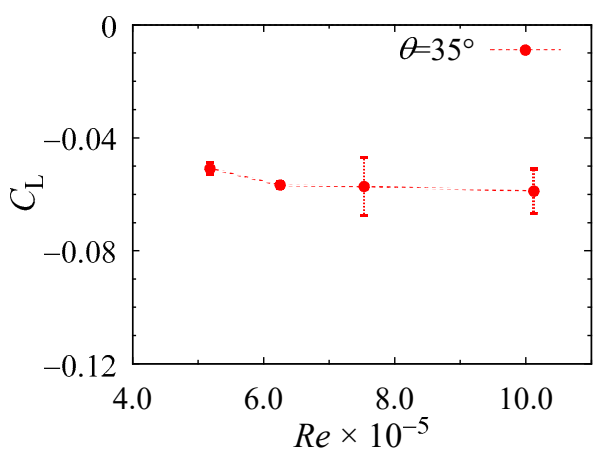

(b)

Figure 9. Towing speed dependency of fluid-dynamic coefficients of model with $\theta=35^{\circ}$ in SMT: (a) dependency of drag coefficient $C_{\mathrm{D}}$ compared with fitted curve for model with $\theta=25^{\circ}$ derived by Meile et al. [4] and (b) dependency of lift coefficient $C_{\mathrm{L}}$.

\subsubsection{Comparison of Cornering Effect with the Previous Numerical Result}

The measurement results of the model with $\theta=25^{\circ}$ were compared with the results of a large eddy simulation at $R e=1.70 \times 10^{6}$ conducted by Keogh et al. [13], and the present measurement result for the fluid-dynamic force in the CMT was validated. To eliminate the difference in friction drag due to the difference in the Reynolds number condition, not the absolute values of the fluid-dynamic coefficients but their changes due to the cornering motion were compared. Figures 10 and 11 show comparisons of the changes in the fluid-dynamic coefficients, $\Delta C_{D}, \Delta C_{L}, \Delta C_{S}$, and $\Delta C_{Y M}$. The changes in the drag, side force, and yaw moment show quantitatively good agreement with the numerical results. In particular, the nonlinear drag increase from $\omega^{\prime}=0.05$ to $\omega^{\prime}=0.1$ was well captured. The lift coefficient was approximately constant, and it also included the numerical result within the confidence interval at $\omega^{\prime}=0.10$. These comparisons validated using the present measurement method to quantitatively measure the changes in the fluid-dynamic coefficients due to the cornering motion.

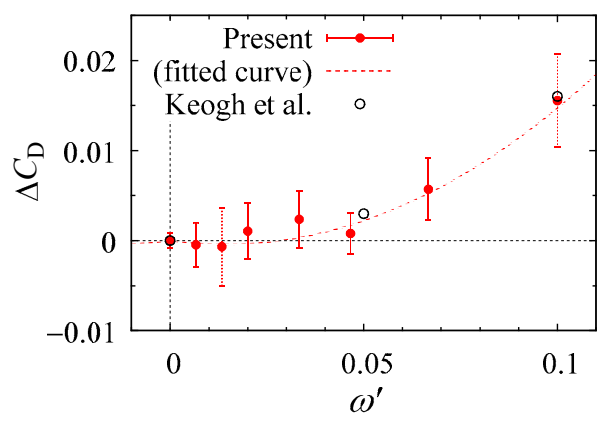

(a)

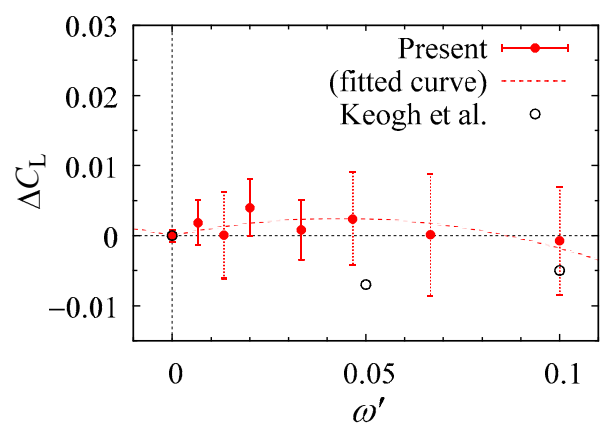

(b)

Figure 10. Comparisons of changes in fluid-dynamic coefficients depending on normalized yaw rate $\omega^{\prime}$ between present measurement at $R e=(6.45 \pm 0.06) \times 10^{5}$ and previous numerical analysis result by Keogh et al. [13] at $R e=1.70 \times 10^{6}:$ (a) change in drag coefficient $\Delta C_{\mathrm{D}} ;(\mathbf{b})$ change in lift coefficient $\Delta C_{\mathrm{L}}$. 


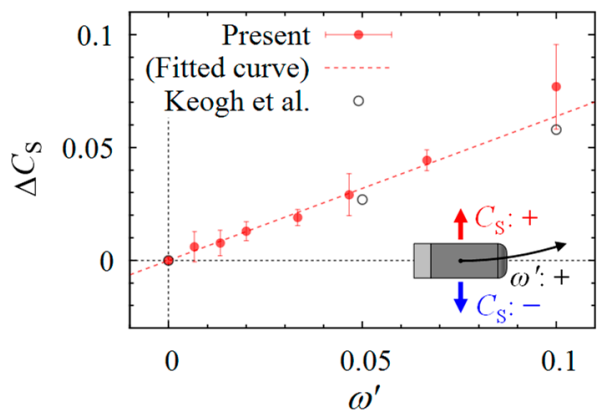

(a)

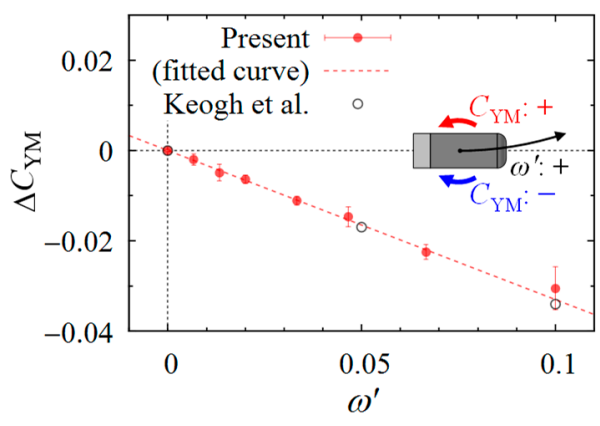

(b)

Figure 11. Comparisons of changes in fluid-dynamic coefficients depending on normalized yaw rate $\omega^{\prime}$ between present measurement at $\operatorname{Re}=(6.45 \pm 0.06) \times 10^{5}$ and previous numerical analysis result by Keogh et al. [13] at $R e=1.70 \times 10^{6}$ : (a) change in side-force coefficient $\Delta C_{\mathrm{S}}$; (b) change in yaw-moment coefficients $\Delta C_{Y M}$.

\subsection{Results of CMT}

The fluid-dynamic forces acting on the two Ahmed models with $\theta=25^{\circ}$ and $\theta=35^{\circ}$ during steady-state cornering were measured, and their characteristics were clarified. Figure 12 shows the dependencies of the $C_{\mathrm{D}}, C_{\mathrm{L}}, \Delta C_{\mathrm{S}}$, and $\Delta C_{\mathrm{YM}}$ values of the two models on normalized yaw rate $\omega^{\prime}$ in the CMT. For all the coefficients, the two models showed qualitatively similar trends. The $C_{\mathrm{D}}$ value increased quadratically, and $C_{\mathrm{L}}$ was approximately constant. At positive yaw rate $\omega^{\prime}>0$, both models showed positive values for $\Delta C_{S}$, which represented the centripetal force of the cornering, and negative values for $\Delta C_{Y M}$, which represented a moment suppressing the yaw rotation.

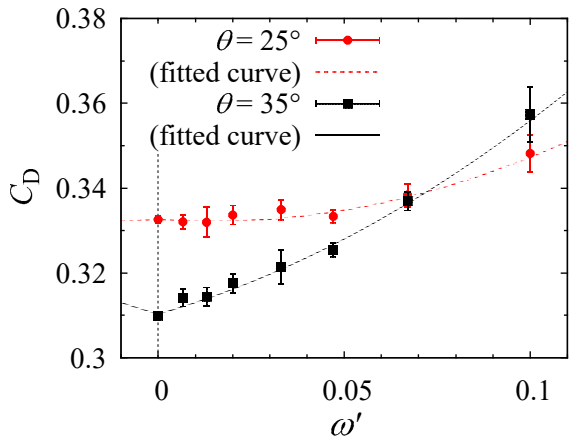

(a)

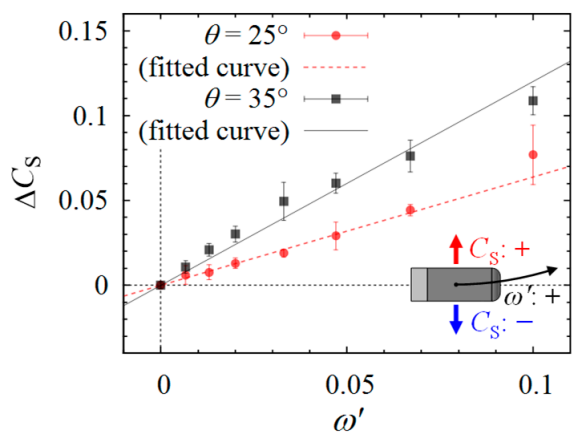

(c)

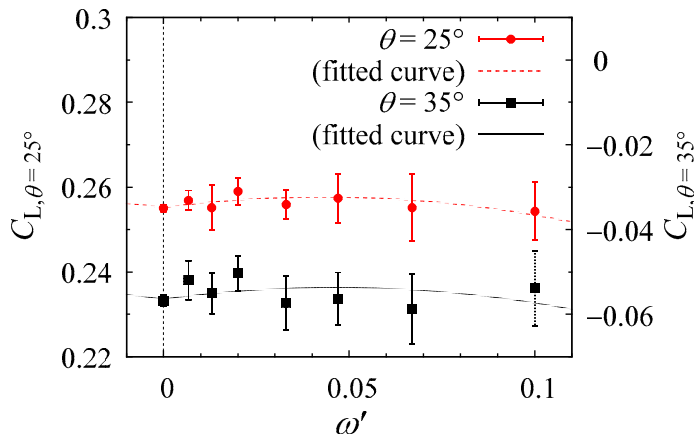

(b)

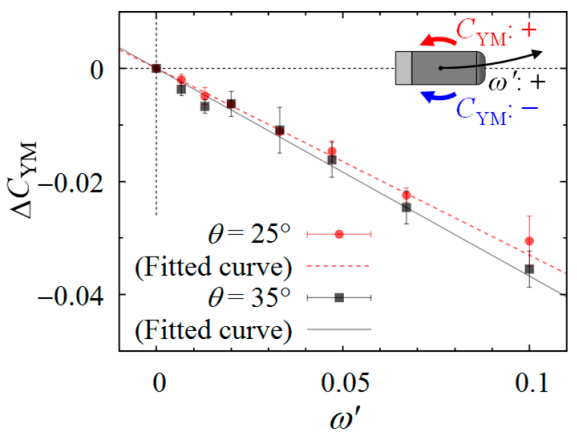

(d)

Figure 12. Comparisons of changes in fluid-dynamic coefficients depending on normalized yaw rate $\omega^{\prime}$ between present measurements: (a) drag coefficient $C_{\mathrm{D}}$; (b) lift coefficient $C_{\mathrm{L}}$; (c) change in side-force coefficient $\Delta C_{S}$; and (d) changes in yaw-moment coefficient $\Delta C_{Y M}$.

In a quantitative comparison between the two models, the model with $\theta=35^{\circ}$ showed a greater drag increase due to cornering than the model with $\theta=25^{\circ}$. The increase in drag coefficient $\Delta C_{D}$ 
with $\theta=35^{\circ}$ reached 0.048 at $\omega^{\prime}=0.10$, while $\Delta C_{D}=0.016$ in the model with $\theta=25^{\circ}$ under the same conditions. They correspond to $15 \%$ and $4.7 \%$ of the total drag in the SMT, respectively. The lift forces of the two models showed similar profiles for the fitted curves, while the absolute values were approximately 0.3 different. The model with $\theta=35^{\circ}$ also showed a greater centripetal side force and yaw moment opposing the yaw rotation than the model with $\theta=25^{\circ}$. In the model with $\theta=35^{\circ}$, the absolute values of the gradients for $\Delta C_{S}$ and $\Delta C_{Y M}$ were $63 \%$ and $11 \%$ greater than those of the model with $\theta=25^{\circ}$.

\subsection{Results of SCW}

The fluid-dynamic forces acting on the Ahmed models with $\theta=25^{\circ}$ and $\theta=35^{\circ}$ under a uniform crosswind condition were reproduced by the SCW and measured as a reference for the discussion on the effect of the spatially distributed crosswind in the CMT. Figure 13 shows the yaw angle dependencies of $C_{\mathrm{D}}, C_{\mathrm{L}}, \Delta C_{\mathrm{S}}$, and $\Delta C_{\mathrm{YM}}$ in the SCW. The $C_{\mathrm{D}}$ value of the model with $\theta=35^{\circ}$ increased with $\beta$, although the $C_{\mathrm{D}}$ value of the model with $\theta=25^{\circ}$ slightly decreased in the measurement range. For the other coefficients, the two models showed the same trend of increasing $C_{\mathrm{L}}$, and decreasing $\Delta C_{\mathrm{S}}$ and $\Delta C_{\mathrm{YM}}$, as $\beta$ increased. These trends for $\Delta C_{\mathrm{S}}$ and $\Delta C_{\mathrm{YM}}$ were general and indicated that the lateral force on the leeward side and the yaw moment turned the head to the leeward side. They showed a non-linear behavior and large variation at $\beta>2.0^{\circ}$, especially for $\Delta C_{\mathrm{S}}$. The model with $\theta=35^{\circ}$ showed a larger absolute value for the gradient of $\Delta C_{\mathrm{S}}$ and a smaller absolute value for $\Delta C_{\mathrm{YM}}$ than the model with $\theta=25^{\circ}$.

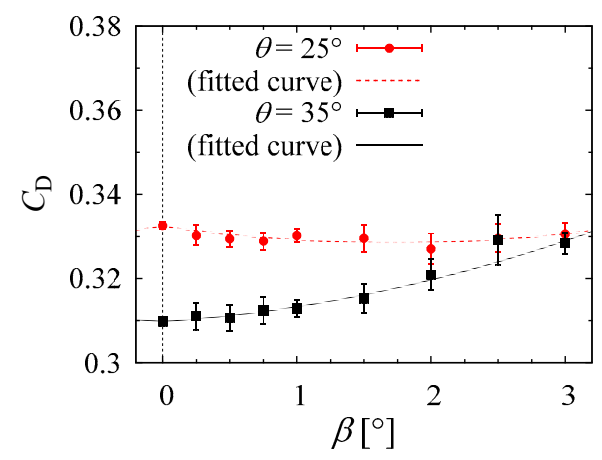

(a)

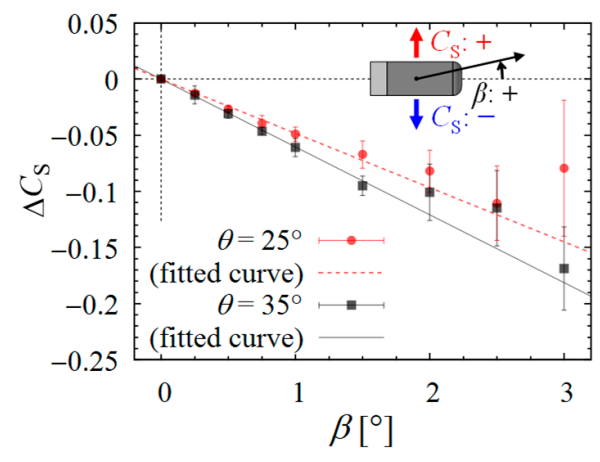

(c)

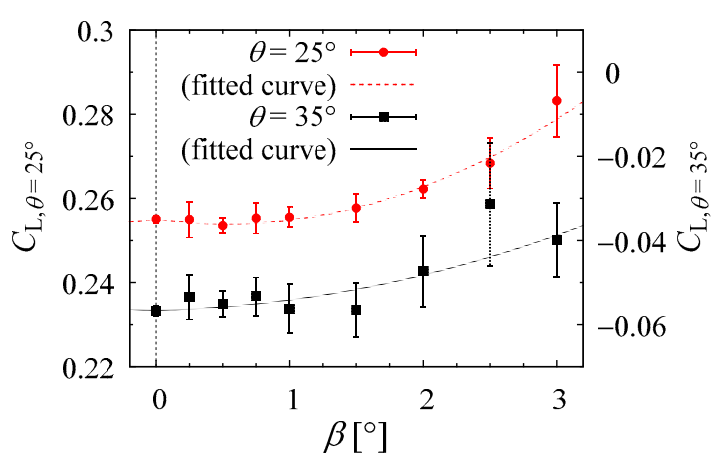

(b)

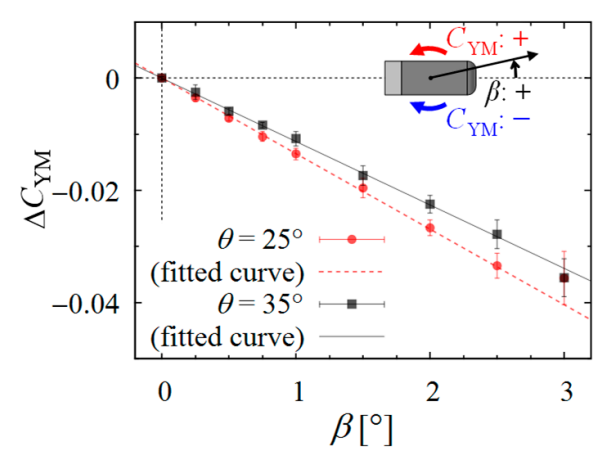

(d)

Figure 13. Comparisons of changes in fluid-dynamic coefficients depending on yaw angle $\beta$ of uniform free-stream between present measurements: (a) drag coefficient $C_{\mathrm{D}} ;(\mathbf{b})$ lift coefficients $C_{\mathrm{L}}$; (c) change in side-force coefficient $\Delta C_{\mathrm{S}} ;(\mathbf{d})$ change in yaw-moment coefficient $\Delta C_{\mathrm{YM}}$. 


\section{Discussion}

Based on the CMT results shown in Section 3.2, the model with $\theta=35^{\circ}$, simulating the wake of a square-back type automobile, caused a larger drag increase due to cornering than the model with $\theta=25^{\circ}$, simulating the wake of a fastback-type automobile. The model with $\theta=35^{\circ}$ showed a drag increase of $15 \%$ at $\omega^{\prime}=0.10$, which represented a corner with a constant radius of 10 times the vehicle length. This drag increase corresponded to approximately $80 \%$ of the drag increase in the model with $\theta=25^{\circ}$ at a corner with a radius of five times the vehicle length, as predicted by Keogh et al. [13]. Because a corner with a larger radius has a higher encounter probability on the road and can be traversed at higher speeds, the drag increase at a corner with a larger radius has a greater impact on the average drag on the road. Therefore, the drag increase of the model with $\theta=35^{\circ}$ in the present results indicated that the drag increase due to cornering motion becomes more important depending on the type of automobile and wake structure.

The changes in the side force and yaw moment due to cornering motion were also greater with $\theta=35^{\circ}$ than with $\theta=25^{\circ}$. Here, the side force acted toward the center of the corner, and the yaw moment acted in the opposite direction to the yaw motion. Thus, the aerodynamic center of the pressure was behind the center of the vehicle. When an external centripetal force acts on a cornering vehicle at a point behind the "center of gravity" $(\mathrm{CoG})$ of the vehicle, the force enhances the understeer characteristics of the vehicle and the stability of its cornering motion. This aerodynamic stabilizing effect depends on both the strength of the centripetal force and the backward distance from the CoG to the force application point. Based on the differences in the side force and yaw moment characteristics between the models with $\theta=25^{\circ}$ and $\theta=35^{\circ}$, the centripetal side force of the model with $\theta=35^{\circ}$ was greater than that of the model with $\theta=25^{\circ}$, while the force application point of the model with $\theta=35^{\circ}$ was further forward than that of the model with $\theta=25^{\circ}$. Therefore, the relationship between the stabilizing effects of the models with $\theta=25^{\circ}$ and $\theta=35^{\circ}$ on the cornering motion depended on the location of the CoG.

Based on the SCW results shown in Section 3.3, the models with $\theta=25^{\circ}$ and $\theta=35^{\circ}$ qualitatively reproduced the relationship of changes in drag, side force and yaw moment between the fast-back type and square-back type automobiles, respectively. The model with $\theta=35^{\circ}$ showed greater increase of drag and side force magnitude, and smaller increase of yaw moment magnitude than the model with $\theta=25^{\circ}$. This tendency corresponded to the general one that the yaw angle dependency of the square-back type automobile has larger changes in drag and side force and smaller change in yaw moment than the yaw angle dependency of the fast-back type automobile [18].

From another point of view, the effects of the crosswind components distributed in the space due to a cornering motion were investigated. The two models had different wake structures and aerodynamic characteristics depending on the slant angle at the rear end of the body. Therefore, the relative flow direction acting on the rear end of the vehicle was considered. The dependency of the fluid-dynamic force on local yaw angle $\beta_{\mathrm{TE}}$ is used for the discussion. Figure 14 shows the change in the aerodynamic force measured by the CMT and SCW with respect to $\beta_{\mathrm{TE}}$. For simplicity, only the fitted curves for each aerodynamic force in Figures 12 and 13 are shown in the graphs. Here, the approximate curve of the CMT result was converted using the relationship between $\beta_{\mathrm{TE}}$ and $\omega^{\prime}$ in Equation (3). The comparison based on the $\beta_{\mathrm{TE}}$ values also shows that the drag increase due to a cornering motion is greater than the drag increase in a uniform crosswind, while the qualitative differences in the drag increase values between the models with $\theta=25^{\circ}$ and $\theta=35^{\circ}$ are consistent in the CMT and SCW. Our previous study [26] discussed the physical mechanisms generating the fluid-dynamic centripetal force due to the cornering motion and indicated that the circulation directly applied to the vehicle body by the yaw rotation generates the centripetal force due to the Magnus effect. This phenomenon would also contribute to the drag increase as its induced drag, which could be one of the reasons that the drag increase in the CMT was greater than that in the SCW.

The lift force shown in Figure 14b only increased under a uniform crosswind condition. However, this difference was caused by a different phenomenon above the top surface of the model. Under uniform 
crosswind conditions, a longitudinal vortex above the top surface is formed starting at the front of the windward edge of the surface [36]. This vertical vortex created negative pressure on the roof, which may have contributed to the increased lift. On the other hand, the cornering motion, where the wind direction is distributed, does not generate such a vortex [13] because the local yaw angle at the center of the model is always approximately zero, and the lift did not increase.

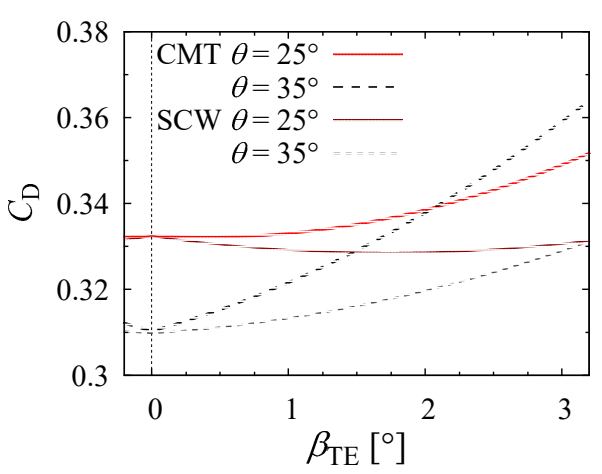

(a)

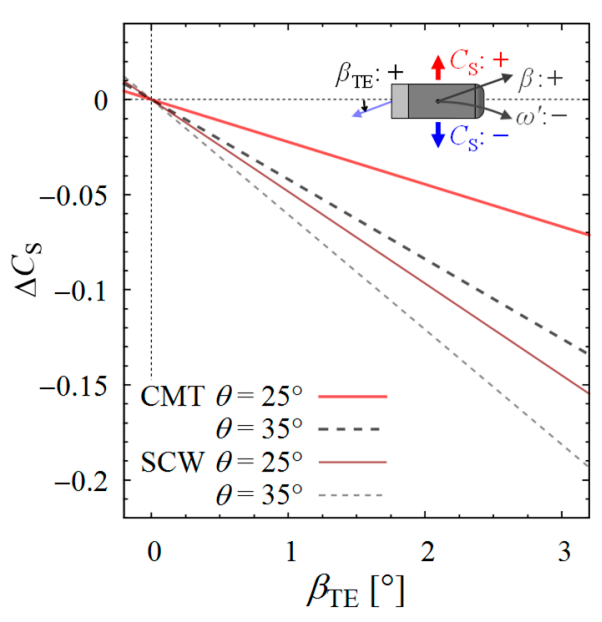

(c)

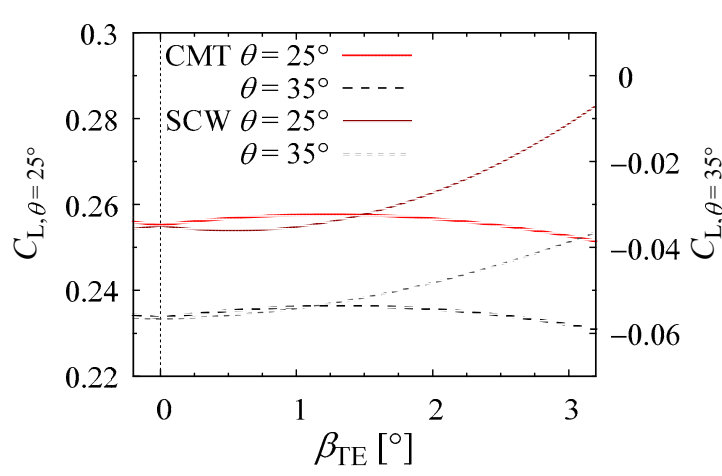

(b)

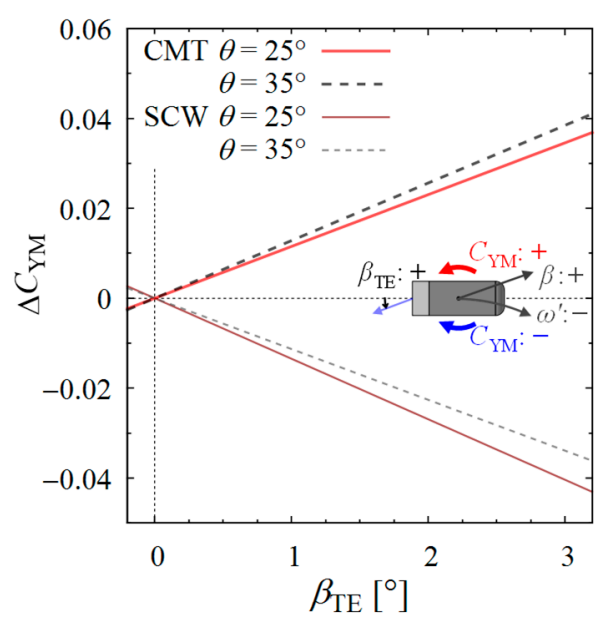

(d)

Figure 14. Comparisons of changes in fluid-dynamic coefficients depending on local yaw angle $\beta_{\mathrm{TE}}$ at the rear end of the model between the CMT and SCW: (a) drag coefficient $C_{\mathrm{D}}$; (b) lift coefficient $C_{\mathrm{L}}$; (c) change in side-force coefficient $\Delta C_{\mathrm{S}} ;$ (d) change in yaw-moment coefficient $\Delta C_{\mathrm{YM}}$.

Regarding the side force and yaw moment shown in Figure 14c,d, consider the local fluid-dynamic response at the rear part of the model to local yaw angle $\beta_{\mathrm{TE}}$. Figure 15 shows a schematic diagram that can be referenced for the following discussion. Because $\beta_{\mathrm{TE}}$ becomes negative during steady-state cornering with a positive yaw rate, and the inside of the corner becomes the leeward side, it can be assumed that the local side force acting on the rear part of the model acts toward the inside of the corner. From the relationship between the changes in the fluid-dynamic forces due to cornering of the models with $\theta=25^{\circ}$ and $\theta=35^{\circ}$, the model with $\theta=35^{\circ}$ shows a greater side force toward the center of the corner than the model with $\theta=25^{\circ}$. The model with $\theta=35^{\circ}$ also shows a greater yaw moment in the opposite direction of the yaw rotation than the model with $\theta=25^{\circ}$. Therefore, the local fluid-dynamic side force acting on the model with $\theta=35^{\circ}$ toward the inside of the corner can be estimated to be greater than that for the model with $\theta=25^{\circ}$. Under a uniform crosswind condition, it can also be assumed that the side force at the rear part acts toward the leeward side and generates a yaw moment that rotates the head toward the windward direction. This increases the absolute value of $\Delta C_{S}$ and 
decreases the absolute value of $\Delta C_{Y M}$ in the SCW results. Because the model with $\theta=35^{\circ}$ shows a larger $\left|\Delta C_{S}\right|$ value and smaller $\left|\Delta C_{Y M}\right|$ value than the model with $\theta=25^{\circ}$, the local fluid-dynamic side force acting on the rear part of the model with $\theta=35^{\circ}$ can be estimated to be greater than that for the model with $\theta=25^{\circ}$. These estimations in CMT and SCW results were consistent with each other. Furthermore, considering that the drag increase of the model with $\theta=35^{\circ}$ is also greater than that of the model with $\theta=25^{\circ}$, the model with $\theta=35^{\circ}$ can be considered to generate a fluid-dynamic force with a greater magnitude at the rear part in response to the local yaw angle than the model with $\theta=25^{\circ}$.
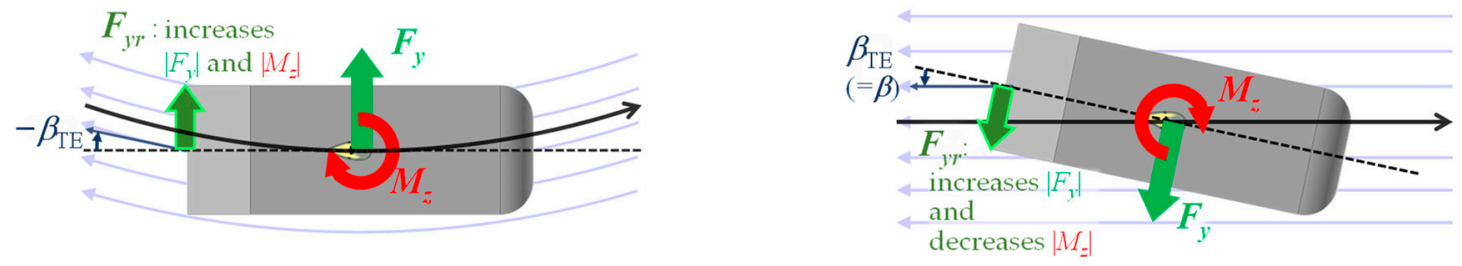

$$
\begin{aligned}
& \left|F_{y}\right|: \theta=25^{\circ}<\theta=35^{\circ} \\
& \left|M_{z}\right|: \theta=25^{\circ}<\theta=35^{\circ}
\end{aligned}
$$

\section{Steady-state cornering} $\left|F_{y r}\right|: \theta=25^{\circ}<\theta=35^{\circ}$
The local side force acting on
the rear part toward the leeward of the local wind direction

$$
\begin{aligned}
& \left|F_{y}\right|: \theta=25^{\circ}<\theta=35^{\circ} \\
& \left|M_{z}\right|: \theta=25^{\circ}>\theta=35^{\circ}
\end{aligned}
$$

Steady crosswind

Figure 15. Schematic image for discussion of side force and yaw moment. The dependency of the side force component of the local fluid-dynamic force acting on the rear part of the model on slant angle $\theta$ is assumed to be consistent in both the CMT and SCW results and the estimation.

\section{Conclusions}

Experimental measurements of the fluid-dynamic forces acting on cornering Ahmed models with different wake characteristics were conducted in a towing tank facility. The increase in drag due to the cornering motion, which was indicated by a previous numerical analysis by Keogh et al. [13], was experimentally captured. Furthermore, the Ahmed model with a slant angle of $\theta=35^{\circ}$, which had a quasi-two-dimensional separated wake, showed a greater drag increase due to the steady-state cornering motion than the model with $\theta=25^{\circ}$, which had a highly three-dimensional wake structure. The drag increase of the model with $\theta=35^{\circ}$ reached $15 \%$ when the cornering radius was 10 times the vehicle length, while the model with $\theta=25^{\circ}$ showed a $5 \%$ drag increase under the same cornering condition. Therefore, it will be more important to consider the cornering effects on the automobile aerodynamics during the development process depending on the type of automobile and its wake characteristics.

When a consistent response is assumed for the local fluid-dynamic characteristics in the local flow direction at the rear end of the body, it is possible to qualitatively explain the relationship between the differences in the drag, side force, and yaw moment from the differences in the geometry and motion of the model. However, the amount of drag increase under the steady-state cornering condition was greater than that under the steady crosswind condition in a comparison based on the local yaw angle defined at the rear end of the model. Therefore, in the quantitative estimation of automobile aerodynamics under realistic on-road conditions, the cornering effects must be precisely reproduced and considered.

Author Contributions: Conceptualization, T.N. and M.T.; methodology, T.N.; software, T.N.; validation, T.N.; formal analysis, T.N.; investigation, T.N.; resources, T.N., H.M., and T.K.; data curation, T.N.; writing-original draft preparation, T.N.; writing—review and editing, T.N., H.M., and T.K.; visualization, T.N.; supervision, H.M. and M.T.; project administration, T.N.; funding acquisition, T.N. All authors have read and agreed to the published version of the manuscript.

Funding: This research was funded by JSPS-KAKENHI (grant numbers 26420115, 17K06159, and 20K04286).

Conflicts of Interest: The authors declare no conflict of interest. 


\section{References}

1. Tunay, T.; Firat, E.; Sahin, B. Experimental investigation of the flow around a simplified ground vehicle under effects of the steady crosswind. Int. J. Heat Fluid Flow 2018, 71, 137-152. [CrossRef]

2. Bonitz, S.; Larsson, L.; Sebben, S. Unsteady pressure analysis of the near wall flow downstream of the front wheel of a passenger car under yaw conditions. Int. J. Heat Fluid Flow 2018, 73, 188-198. [CrossRef]

3. Guilmineau, E.; Chikhaoui, O.; Deng, G.; Visonneau, M. Cross wind effects on a simplified car model by a DES approach. Comput. Fluids 2013, 78, 29-40. [CrossRef]

4. Meile, W.; Ladinek, T.; Brenn, G.; Reppenhagen, A.; Fuchs, A. Non-symmetric bi-stable flow around the Ahmed body. Int. J. Heat Fluid Flow 2016, 57, 34-47. [CrossRef]

5. Wieser, D.; Vayeri, C.N.; Paschereit, C.O. Wake structures and surface patterns of the DrivAer Notchback car model under side wind conditions. Energies 2020, 13, 320. [CrossRef]

6. Wordley, S.; Saunders, J. On-road turbulence. SAE Int. J. Passeng. Cars Mech. Syst. 2009, 1, 341-360. [CrossRef]

7. Wordley, S.; Saunders, J. On-road turbulence: Part 2. SAE Int. J. Passeng. Cars Mech. Syst. 2009, 2, 111-137. [CrossRef]

8. Carlino, G.; Cogotti, A. Simulation of Transient Phenomena with the Turbulence Generation System in the Pininfarina Wind Tunnel; SAE Technical Paper; SAE International: Warrendale, PA, USA, 2006. [CrossRef]

9. Carlino, G.; Cardano, D.; Cogotti, A. A New Technique to Measure the Aerodynamic Response of Passenger Cars by a Continuous Flow Yawing; SAE Technical Paper; SAE International: Warrendale, PA, USA, 2007. [CrossRef]

10. Stoll, D.; Schoenleber, C.; Wittmeier, F.; Kuthada, T.; Wiediemann, J. Investigation of Aerodynamic Drag in Turbulent Flow Conditions. SAE Int. J. Passeng. Cars Mech. Syst. 2016, 9, 733-742. [CrossRef]

11. Shimizu, K.; Nakashima, T.; Hiraoka, T.; Nakamura, Y.; Nouzawa, T.; Doi, Y. Aerodynamic drag change of simplified automobile models influenced by a passing vehicle. Mech. Eng. J. 2020, 7, 19-00366. [CrossRef]

12. Keogh, J.; Doig, G.; Diasinos, S.; Barber, T. The influence of cornering on the vortical wake structures of an inverted wing. Proc. Inst. Mech. Eng. D J. Automob. Eng. 2015, 229, 1817-1829. [CrossRef]

13. Keogh, J.; Barber, T.; Diasinos, S.; Doig, G. The aerodynamic effects on a cornering Ahmed body. J. Wind Eng. Ind. Aerodyn. 2016, 154, 34-46. [CrossRef]

14. Josefsson, E.; Hagvall, R.; Urquhart, M.; Sebben, S. Numerical Analysis of Aerodynamic Impact on Passenger Vehicles during Cornering; SAE Technical Paper; SAE International: Warrendale, PA, USA, 2018. [CrossRef]

15. Tsubokura, M.; Ikawa, Y.; Nakashima, T.; Okada, Y.; Kamioka, T.; Nouzawa, T. Unsteady vehicle aerodynamics during a dynamic steering action: 2nd report, numerical analysis. SAE Int. J. Passeng. Cars Mech. Syst. 2012, 5, 340-357. [CrossRef]

16. Okada, Y.; Nakashima, T.; Tsubokura, M.; Morikawa, Y.; Kouno, R.; Okamoto, S.; Nouzawa, T. Aerodynamics Evaluation of Road Vehicles in Dynamic Maneuvering; SAE Technical Paper; SAE International: Warrendale, PA, USA, 2016. [CrossRef]

17. Keogh, J.; Barber, T.; Diasinos, S.; Doig, G. Techniques for Aerodynamic Analysis of Cornering Vehicles; SAE Technical Paper; SAE International: Warrendale, PA, USA, 2015. [CrossRef]

18. Schuetz, T. Aerodynamics of Road Vehicles, 5th ed.; SAE International: Warrendale, PA, USA, 2015; pp. $282-290$.

19. Gordes, A. Process for Simulating Curved Airflows on Wheeled Vehicles in Fluid Flow Channels with a Straight Measuring Section. Patent No. EP1610111A2, 28 December 2005.

20. Keogh, J.; Barber, T.; Diasinos, S.; Doig, G. A New Type of Wind Tunnel for the Evaluation of Curved Motion. In Proceedings of the 54th AIAA Aerospace Sciences Meeting, San Diego, CA, USA, 4-8 January 2016; No. AIAA 2016-1774. [CrossRef]

21. Ueno, M.; Yoshimura, Y.; Tsukada, Y.; Miyazaki, H. Circular motion tests and uncertainty analysis for ship maneuverability. J. Mar. Sci. Technol. 2009, 14, 469. [CrossRef]

22. Vorwaller, M.L.; Germane, G.J. Aerodynamic Drag Studies on Rolling Vehicles by Underwater Tow Testing; SAE Technical Paper; SAE International: Warrendale, PA, USA, 1986. [CrossRef]

23. Larsson, L.; Hammar, L.; Nilsson, L.U.; Berndtsson, A.; Knutson, K.; Danielson, H. A Study of Ground Simulation Correlation between Wind Tunnel and Water-Basin Tests of a Full-Scale Car; SAE Technical Paper; SAE International: Warrendale, PA, USA, 1989. [CrossRef]

24. Aoki, K.; Miyata, H.; Kanai, M.; Hanaoka, Y.; Zhu, M. A Water-Basin Test Technique for the Aerodynamic Design of Road Vehicles; SAE Technical Paper; SAE International: Warrendale, PA, USA, 1992. [CrossRef] 
25. Ogawa, A.; Mashio, S.; Yano, S.; Kawamura, T. Quantitative representations of aerodynamic effects on handling response and flat ride of vehicles. SAE Int. J. Passeng. Cars Mech. Syst. 2012, 5, 304-323. [CrossRef]

26. Nakashima, T.; Tsubokura, M.; Okada, Y.; Nouzawa, T.; Mizokane, M.; Doi, Y. Aerodynamic force acting on a road vehicle in steady-state cornering. Trans. JSME (in Japanese) 2014, 80, FE0301. [CrossRef]

27. Nakashima, T.; Tsubokura, M.; Okada, Y.; Nouzawa, T.; Kono, R.; Doi, Y. Aerodynamic Characteristics of a Road Vehicle in Steady-State Cornering. In Proceedings of the ASME/JSME/KSME 2015 Joint Fluids Engineering Conference, Seoul, Korea, 26-31 July 2015; V01AT17A002. [CrossRef]

28. Ahmed, S.; Ramm, G.; Faltin, G. Some Salient Features of the Time-Averaged Ground Vehicle Wake; SAE Technical Paper; SAE International: Warrendale, PA, USA, 1984. [CrossRef]

29. Lienhart, H.; Stoots, C.; Becker, S. Flow and Turbulence Structures in the Wake of a Simplified Car Model (Ahmed Modell). In New Results in Numerical and Experimental Fluid Mechanics III. Notes on Numerical Fluid Mechanics (NNFM); Wagner, S., Rist, U., Heinemann, H.J., Hilbig, R., Eds.; Springer: Berlin/Heidelberg, Germany, 2002; Volume 77. [CrossRef]

30. Kohri, I.; Yamanashi, T.; Nasu, T.; Hashizume, Y.; Katoh, D. Study on the transient behaviour of the vortex structure behind Ahmed body. SAE Int. J. Passeng. Cars Mech. Syst. 2014, 7, 586-602. [CrossRef]

31. Kasai, A.; Shiratori, S.; Kohri, I.; Kobayashi, Y.; Katoh, D.; Nagano, H.; Shimano, K. Large-scale separated vortex generated in a wake flow of Ahmed's body. Flow Turbul. Combust. 2019, 102, 373-388. [CrossRef]

32. Guilmineau, E. Computational study of flow around a simplified car body. J. Wind Eng. Ind. Aerodyn. 2008, 96, 1207-1217. [CrossRef]

33. Guilmineau, E.; Deng, G.B.; Leroyer, A.; Queutey, P.; Visonneau, M.; Wackers, J. Assessment of hybrid RANS-LES formulations for flow simulation around the Ahmed body. Comput. Fluids 2018, 176, 302-319. [CrossRef]

34. Sumida, M.; Hayakawa, K. Aerodynamic forces acting on Ahmed-type vehicles under fluctuating headwind conditions. J. Appl. Fluid Mech. 2019, 12, 1563-1574. [CrossRef]

35. Bae, I.; Moon, J.; Seo, J. Toward a comfortable driving experience for a self-driving shuttle bus. Electronics 2019, 8, 943. [CrossRef]

36. Rao, A.; Minelli, G.; Basara, B.; Krajnović, S. On the two flow states in the wake of a hatchback Ahmed body. J. Wind Eng. Indust. Aerodyn. 2018, 173, 262-278. [CrossRef]

Publisher's Note: MDPI stays neutral with regard to jurisdictional claims in published maps and institutional affiliations.

(C) 2020 by the authors. Licensee MDPI, Basel, Switzerland. This article is an open access article distributed under the terms and conditions of the Creative Commons Attribution (CC BY) license (http://creativecommons.org/licenses/by/4.0/). 